\title{
Multi-agent location system in wireless networks
}

\author{
Luis Mengual, Oscar Marbán *, Santiago Eibe, Ernestina Menasalvas \\ Faculty of Computer Science, Universidad Politécnica de Madrid, Campus de Montegancedo, s/n. 28660 Boadilla del Monte, Madrid, Spain
}

A B S T R A C T

In this paper we propose a flexible Multi-Agent Architecture together with a methodology for indoor location which allows us to locate any mobile station (MS) such as a Laptop, Smartphone, Tablet or a robotic system in an indoor environment using wireless technology. Our technology is complementary to the GPS location finder as it allows us to locate a mobile system in a specific room on a specific floor using the Wi-Fi networks.

The idea is that any MS will have an agent known at a Fuzzy Location Software Agent (FLSA) with a minimum capacity processing at its disposal which collects the power received at different Access Points distributed around the floor and establish its location on a plan of the floor of the building. In order to do so it will have to communicate with the Fuzzy Location Manager Software Agent (FLMSA). The FLMSAs are local agents that form part of the management infrastructure of the Wi-Fi network of the Organiza-tion.

The FLMSA implements a location estimation methodology divided into three phases (measurement, calibration and estimation) for locating mobile stations (MS). Our solution is a fingerprint-based position-ing system that overcomes the problem of the relative effect of doors and walls on signal strength and is independent of the network device manufacturer.

In the measurement phase, our system collects received signal strength indicator (RSSI) measurements from multiple access points. In the calibration phase, our system uses these measurements in a normal-ization process to create a radio map, a database of RSS patterns. Unlike traditional radio mapbased methods, our methodology normalizes RSS measurements collected at different locations on a floor. In the third phase, we use Fuzzy Controllers to locate an MS on the plan of the floor of a building.

Experimental results demonstrate the accuracy of the proposed method. From these results it is clear that the system is highly likely to be able to locate an MS in a room or adjacent room.

\section{Introduction}

The use of wireless technology has expanded over recent years. Universities, private companies, as well as stations and airports all provide wireless communications facilities. This type of network can be implemented using what are known as access-point devices that provide total connectivity over a surface and connection to the Internet. Several such devices are sometimes necessary to cover an office, a university or an airport.

One of the services to be implemented on these networks is user location on the premises. Through the location and the visualization of a plan of the coverage area, users can navigate the premises as they would do using GPS navigation in an outdoor environment. Additionally, a network manager would be able to pinpoint users in these environments and interact with them through their positioning. Indoor positioning technology can also complement mobile-

\footnotetext{
* Corresponding author. Tel.: +34 913367388; fax: +34 913367393.

E-mail addresses: lmengual@fi.upm.es (L. Mengual), omarban@fi.upm.es (O. Marbán), seibe@fi.upm.es (S. Eibe), emenasalvas@fi.upm.es (E. Menasalvas).
}

telephone-based GPS user positioning to determine the user's exact location indoors (Soria, Ortega, Alvarez, \& Gonzalez-Abril, 2012).

In another context, location tracking is an essential feature for enterprises building business-critical wireless networks. If information technology (IT) staff can identify and track the location of wireless clients and highly mobile assets, they can improve the accuracy of WLAN planning and deployment, optimize ongoing network performance, enhance wireless security, and improve the usefulness and value of important business applications. Location tracking provides enhanced visibility and control of air space, helping IT staff to deploy wireless networks that are as easy to manage and as effective to deploy as traditional wired networks.

To address lack-of-visibility problems, organizations need a cost-effective, easy-to-deploy solution for tracking and managing thousands of Wi-Fi devices and tags across a variety of business environments.

In heterogeneous environments, e.g. inside a building, the received power is a very complex function of distance, wall geometry, building infrastructures and obstacles. Even if you have a detailed model of the building, it takes a lengthy simulation to solve the 
direct problem of deriving signal strength given the location. This is what has motivated us to consider flexible models based on function networks (neural networks) to implement a system to locate MSs.

The received signal strength (RSS), a measure of the power received by the client from an access point (AP), is the key parameter for establishing the position of an MS in an indoor environment. Traditionally, WLANs have used different measurement techniques to derive the position of MSs. There are three major categories: closest access point-based, location pattern or fingerprint and distance or angle measurement techniques (see Fig. 1).

The closest AP method finds devices within the total coverage area of a single AP. It is the simplest but least accurate way of locating a device or user. With the closest AP method, the location tracking system only identifies devices within the total coverage area of a single AP. This area can be quite large and include multiple rooms. This system considers an AP to which a terminal connects as the user location.

The main distance measurement-based approach is known as TDOA (Time Difference of Arrival). This technique relies on the timing precision between the signal transmitter and receiver. It uses the propagation delay to calculate the distance between the transmitter and receiver (Junyang, Molisch, \& Salmi, 2012; Zietek, Kolakowski, \& Modelski, 2012). Therefore, a precise synchronization is very important in such systems. By combining at least three distances from three reference positions, triangulation can be used to estimate the MS's location. These techniques will require a highaccuracy clock in the communication system.

Angle of arrival $(A O A)$ is a technique based on angle measurement. This methodology locates the MS by determining the signal's angle of incidence. The location can be by estimated using simple geometric relationships, such as triangulation or geometrical transformation methods (Bo-Chieh \& Ken-Huang, 2008; Genming, Zhenhui, Lingwen, Ziqi, \& Jinbao, 2012).

In indoor environments, however, the distance between transmitter and receiver is usually shorter than the time resolution that the system can measure. Additionally, the MS is surrounded by scattered objects, which results in multiple angles of signal reception. Therefore, the $A O A$ and TDOA approaches are difficult to implement in indoor environments.

Fingerprinting techniques generally only require the measurement of received signal strength or other non-geometric features at several locations to draw up a database of location fingerprints (Del Mundo, Ansay, Festin, \& Ocampo, 2011). To estimate the mobile location, the system first needs to measure the received signal strength at particular locations and then search for the pattern or fingerprints with the closest match in the database.

Generally, the deployment of fingerprinting-based positioning systems can be divided into two phases. First, the location fingerprints are collected in the off-line or calibration phase by carrying out a site survey of the received signal strength (RSS) from multiple

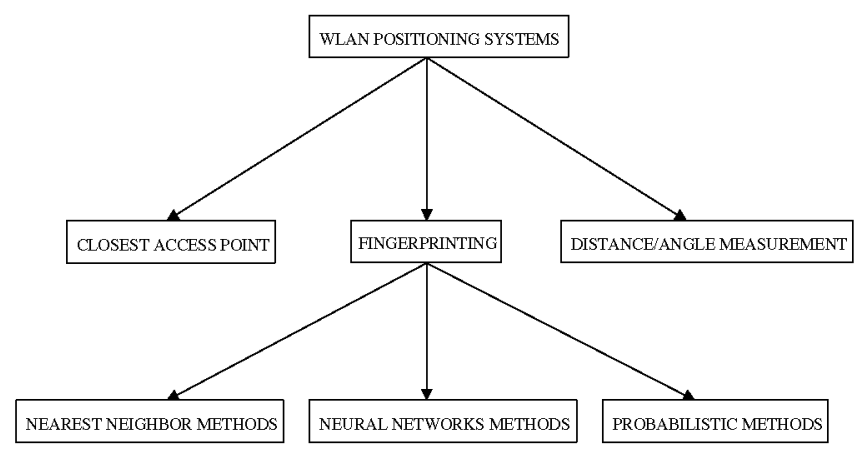

Fig. 1. WLAN positioning techniques.
APs. Enough RSS measurements are taken to set up a database or a table of RSS patterns for predetermined points of the coverage area. The database of RSS patterns with their respective locations is called a radio map.

Second, an MS will report a sample measured vector of RSSs from different APs to a central server (or a group of APs will gather the RSS measurements from an MS and send it to the server) in the on-line or estimation phase. The server uses a positioning algorithm to estimate the location of the MS based on the radio map and reports the estimate back to the MS (or the application requesting the position information).

At this point we have several possibilities. The most common algorithm used to estimate the location computes the Euclidean distance between the sample-measured RSS vector and each fingerprint in the database. For indoor positioning systems, other advanced algorithms and techniques, such as neural networks (Chun-Yu, Lyu-Han, Gen-Huey, Wu, \& Ming-Hui, 2012; Figuera, Rojo-Âlvarez, Wilby, Mora-Jiménez, \& Caamaño, 2012; Mengual, Marbán, \& Eibe, 2010), probabilistic methods (Chai \& Yang, 2007; Chung, Chen, \& Tang, 2012; Kaemarungsi \& Krishnamurthy, 2012; Shum, Ng, \& Quan, 2012) or fuzzy logic (Abdelhadi \& Anan, 2012) have been introduced to determine the relationship between the RSS samples and the location fingerprint on the radio map.

Other approaches have been presented. For example, some authors have proposed simulating the calibration phase with a ray-tracing model (Sawada, Takahashi, \& Kato, 2012). However, this model requires a highly detailed model of the building. It could be tricky, if not unfeasible, to achieve such a detailed model. One of the main drawbacks of both of these models is that signal strength prediction is dependent not just on the layout of the building, but also on the position of many other hard-to-model components, such as furniture, equipment and human beings.

So far, fingerprinting techniques with a real calibration phase have attracted more attention because they are a simple and the most effective solution for indoor environments.

of the solutions that use RSSI to locate a mobile user, some scan the radio-electric spectrum of every square meter of the coverage area. Additionally, they only use one card brand. It is unfeasible to position another mobile user with a different Wi-Fi receiver using this solution.

The solution proposed in this paper is a fingerprint-based positioning system that, integrated into the agent code, gets significantly better results than other similar systems because it normalizes measurements and optimizes clusters formed according to the physical features of the ground plan. In this way, the proposed solution is valid for any $\mathrm{Wi}$-Fi receiver, as we account for the relative effect of walls and obstacles as a relative power increase or decrease.

Additionally, our technique does not require an exhaustive scan of the coverage area. Actually we present the results of sampling just one point per location. Our system can adapt to the geometry of each scenario and operate with any Wi-Fi device (regardless of the manufacturer).

Finally, we present the results of implementing our system in practice and the evaluation tests run in our workplace. The experimental results confirm the effectiveness of our methodology.

\section{Location estimation system issues}

Unfortunately, commercial 802.11 hardware does not provide positioning functionality. Even so, positioning can be implemented using RSS measurements provided by an 802.11 wireless card. RSS is a measure of the power received by the client from an AP and provides information as to the client's location.

In heterogeneous environments, e.g. inside a building, the received power is a very complex function of distance, geometry, 
and materials. This is further complicated by the fact that the signal propagation is influenced by environmental factors such as the number of people in the working area, the position of walls and other building infrastructures and the materials they are made of, as well as the multi-path effect (Ali \& Nobles, 2007).

Another problem is the RSSI gathered from wireless stations using their device driver. The RSSI is an optional parameter that has a value of 0 through to RSSI_Max. This parameter is a measure of the physical sub-layer of the energy observed at the antenna used to receive the current frame. The RSSI shall be measured from the beginning of the start frame delimiter (SFD) to the end of the frame, i.e. header error check (HEC). The RSSI is intended to be used in a relative manner. An absolute RSSI reading accuracy is not specified.

There is nothing in the 802.11 standard that stipulates a relationship between the RSSI value and any particular energy level as could be measured in $\mathrm{mW}$ or $\mathrm{dBm}$. Individual vendors have chosen to provide their own levels of accuracy, granularity, and actual power range (measured as $\mathrm{mW}$ or $\mathrm{dBm}$ ) and their RSSI value range (from 0 to RSSI_Max). Some vendors do not provide an RSSI value and instead convert directly from $\mathrm{dBm}$ to percent.

In this study we have used our own RSS collecting software to test different wireless cards. Each card collected 100 samples (1 sample/s) on the 3rd Floor of Building 4 at the School of Computing (Universidad Politécnica Madrid). Fig. 2 above shows the measurable ranges for the different wireless cards in a room. We found that there was a difference of $20 \mathrm{~dB}$ between different cards (3Com/Ovislink). There is even a card (USRobotics) that implements the RSSI as a percentage and not in $\mathrm{dBm}$ units.

From these results we conclude that the mapping between the actual RF energy and the RSSI range in a wireless device varies from one vendor to another. Since both the range and the measurement of the RSS depend on the WLAN card, most fingerprint-based positioning systems have used the same wireless card vendor to collect the location fingerprints and determine the location.

The results of our experimental tests match the study run in (Kaemarungsi \& Krishnamurthy, 2012), showing the spread of values for five card manufacturers. However, our methodology is capable of working with different wireless cards because we normalize all of the RSS measurements in the calibration phase.

\section{Multi-agent location system}

The system that we propose in this paper is based on a MultiAgent Architecture capable of being adapted to any technology or mobile station (MS). The idea is for the MSs to have a minimum processing capacity agent at its disposal (Fuzzy Location Software Agent). This agent will implement a protocol with the Fuzzy Location Software Manager Agent (FLMSA) with the objective of establishing its exact location of the MS on the floor of the movement agent.

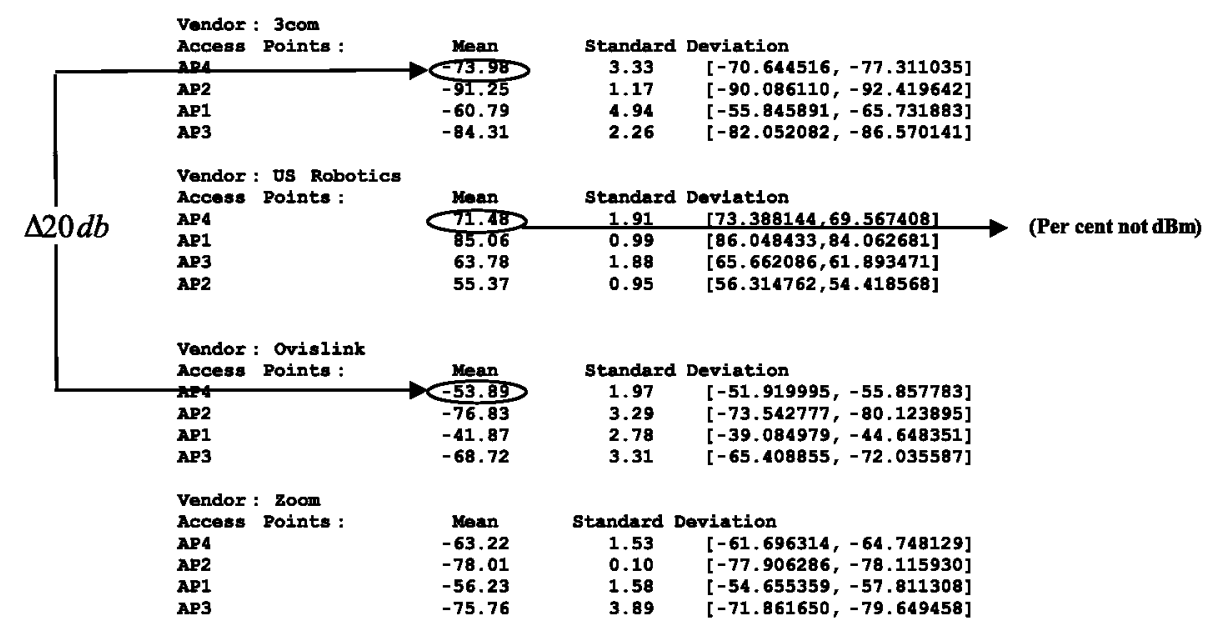

Fig. 2. Measurable RSS range of WLAN cards.

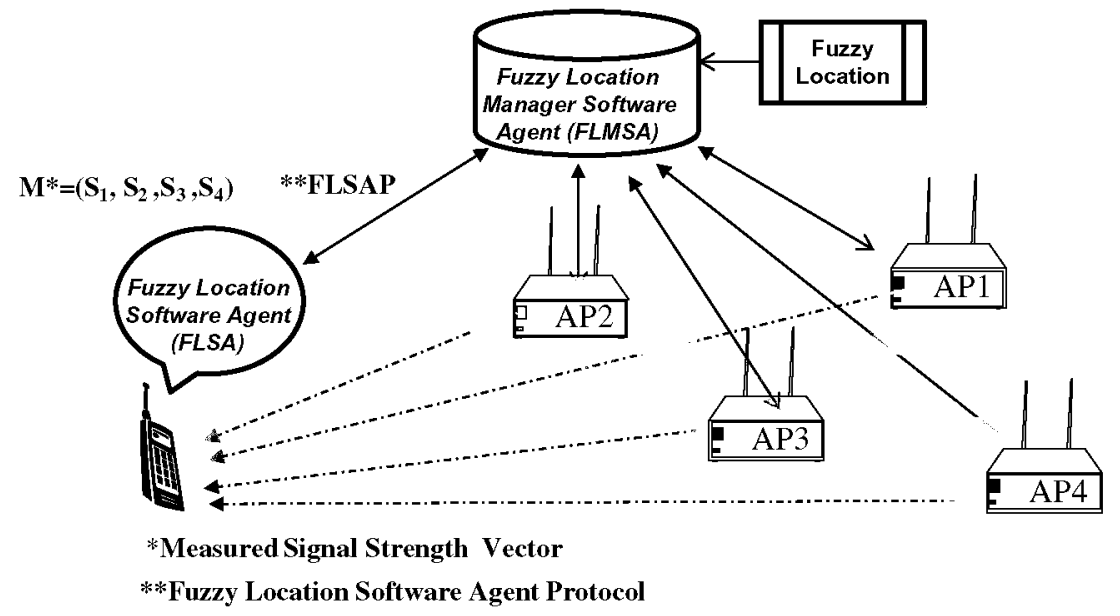

Fig. 3. Multi-agent location system of an MS. 


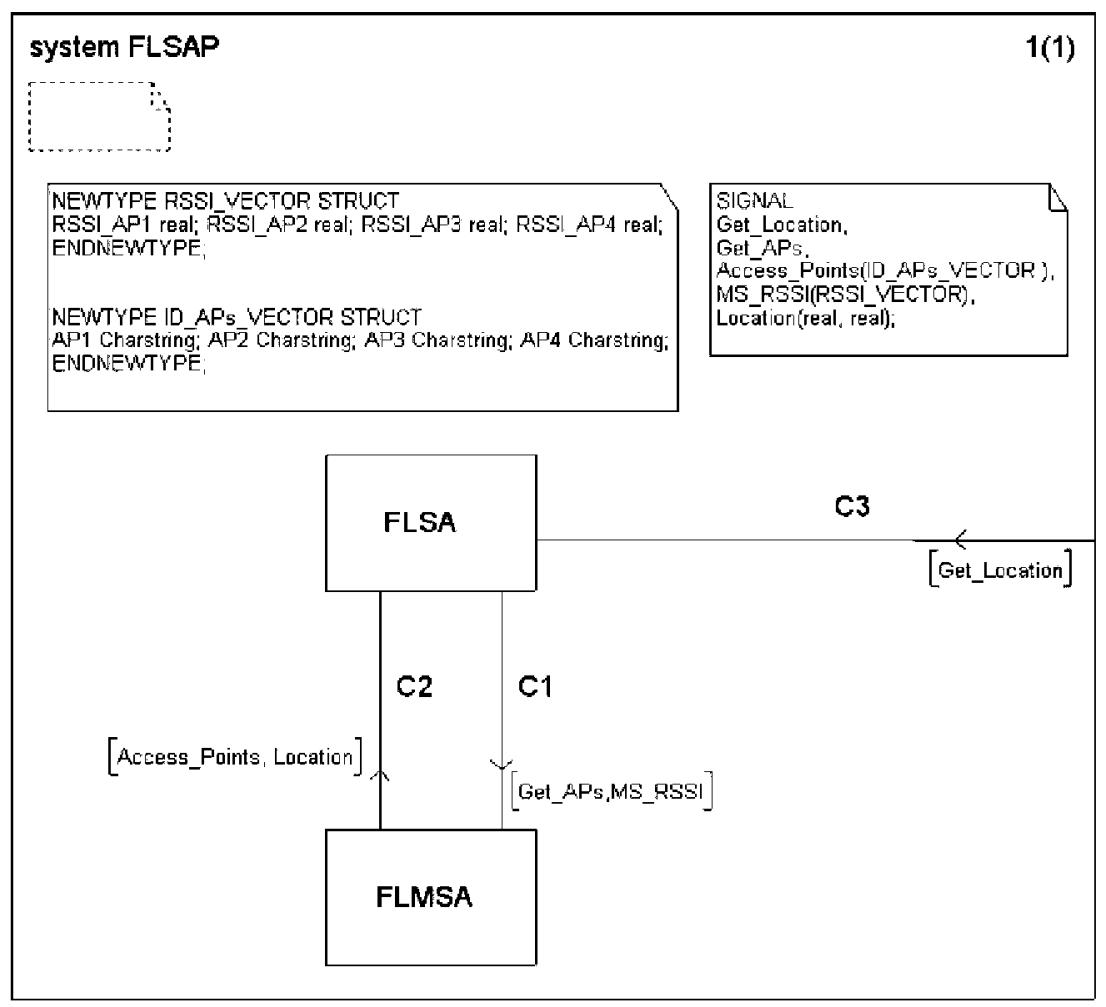

Fig. 4. Description of the level of the system. FLSAP Specification.

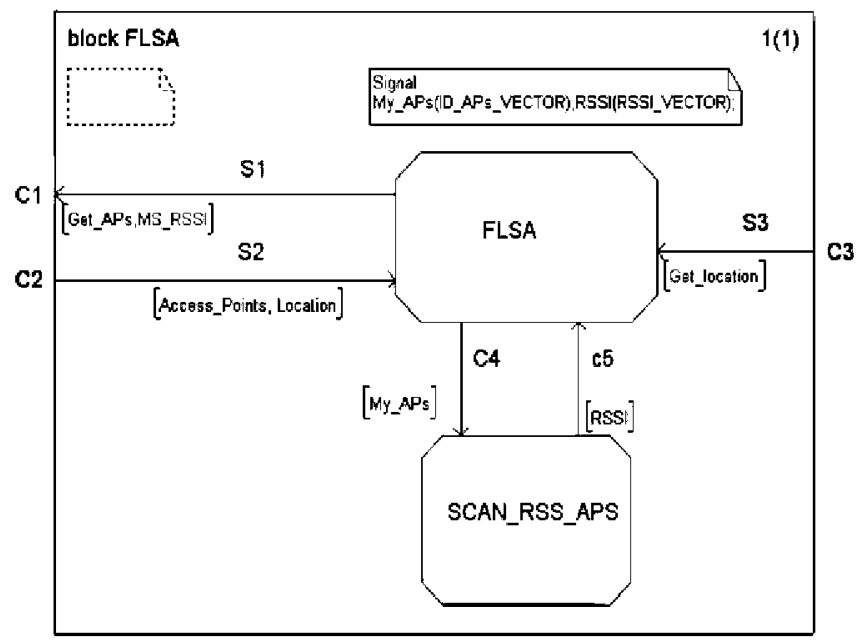

Fig. 5. Description of the block level of the FLSA.

\subsection{Outline of the system}

The complete description of the system is set out in Fig. 3 . The system that we propose in this paper is based on a multi-agent system that allows any mobile MS station, be it a laptop, Smartphone, tablet or a robotic system to be located on the floor of a building. Our location system is complementary to the GPS location system, allowing the MS to be situated in a specific room within a building.

In this paper we propose the standardization of a minimum processing software agent called a Fuzzy Location Software Agent (FLSA) which when installed in an MS is capable of being synchronized by means of a fuzzy location software agent protocol (FLSAP) protocol with a local location agent called a Fuzzy Location Man-

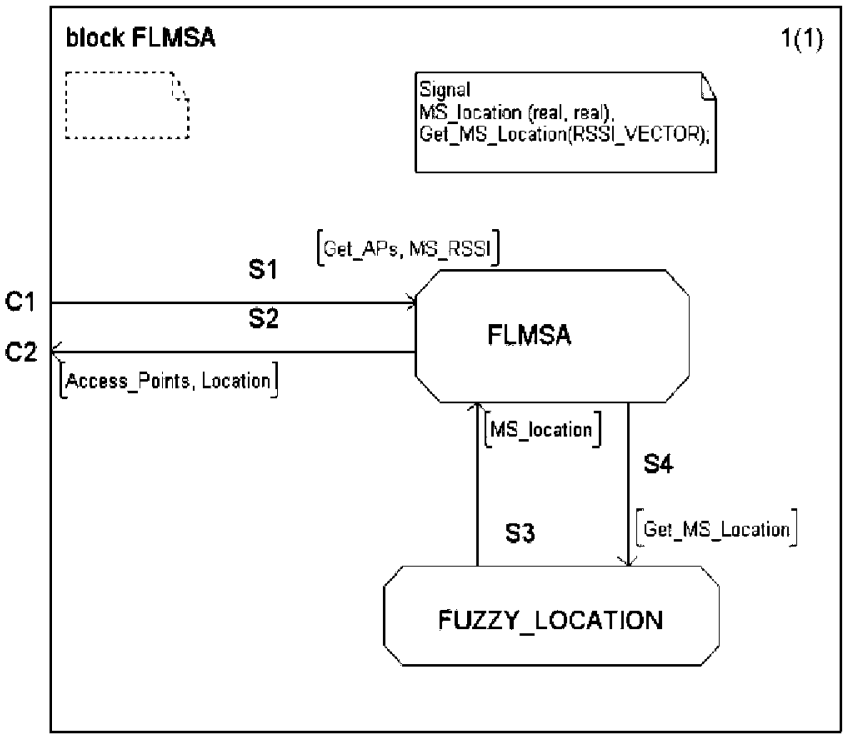

Fig. 6. Description of the block level of the FLMSA.

ager Software Agent (FLMSA) with the objective of obtaining the location of the MS on the floor of a building.

The FLMSAs are local agents that form part of the management infrastructure of the Wi-Fi network of the Organization. If other software agents or modules of the management system are in charge of maintaining the connectivity of the mobile equipment that are distributed around the floor of a building, the FLMSAs are in charge of managing the access points of the floor in order to locate an MS within the radio coverage of the access points.

The process is as follows: the FLMSA's knows the points of access on the floor of a building, as well as the detailed plan of the floor under consideration together with the rooms. 


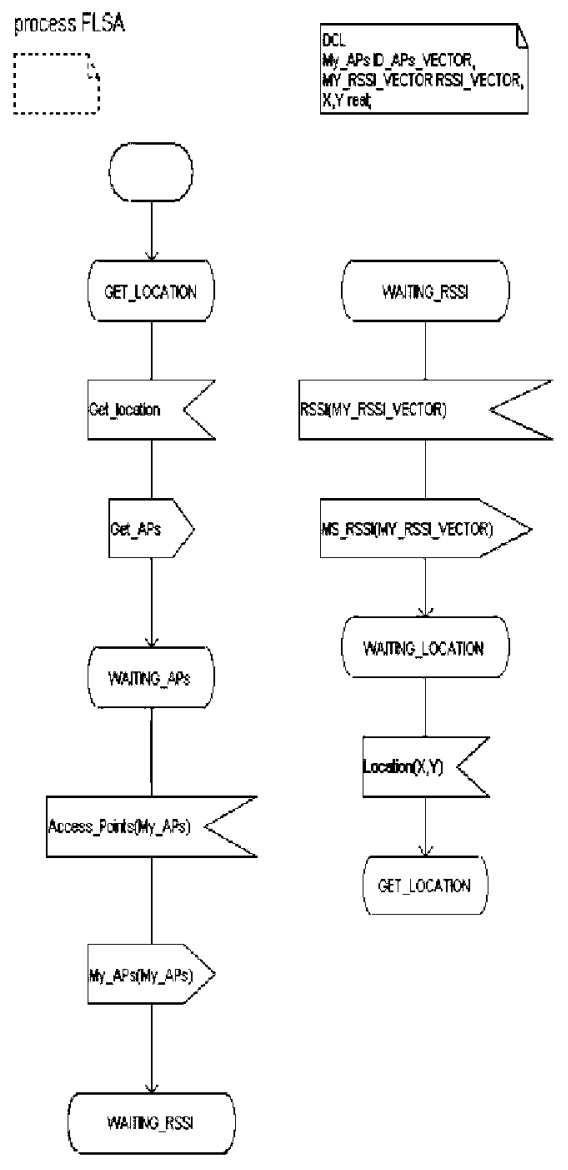

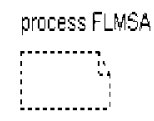

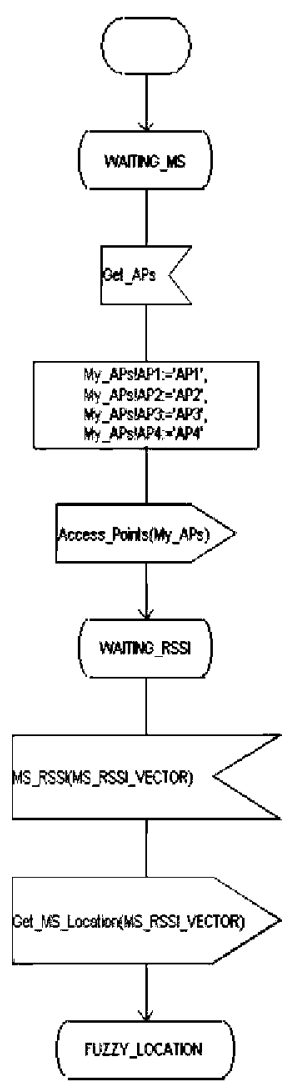

$\mathrm{DCL}$ MS_RST_YECTOR RSS_YYECTOP $x, y$ rest

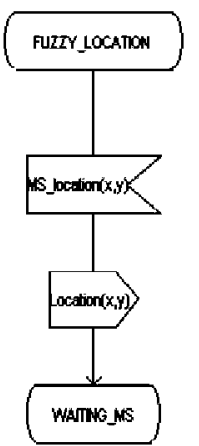

Fig. 7. Description of the process level of the FLMSA

When a mobile station (MS) enters the radio coverage of the access points on the floor, the (FLMSA) is informed of the existence of an MS. The FLMSA sends the MS information on the APs which are used to determine the location of the MS.

Now the MS gathers the RSSI of the access points selected by its Wi-Fi interface and builds a power vector (Measurement Signal Strength Vector). This vector is finally sent to the FLMSA which, by means of an estimation methodology based on fuzzy rules, establishes the location of the MS. This location is sent to the MS and potentially sent to the Internet in order to have the user located. Obviously this option requires permission from the MS.

\subsection{Fuzzy location software agent protocol (FLSAP)}

The fuzzy location software agent protocol is a set of exchanges that are established between the FLSA and the FLMSA with the objective of locating the MS.

In this paper we present the formal specification of this protocol using SDL, Specification and Description Language (SDL and description language ITU-T Rec. Z.100 (11/99)) (Specification and description language ITU-T Rec. Z.100) and the IBM Rational SDL Suite tool (IBM $^{\mathbb{E}}$ Rational $^{\mathbb{B}}$ SDL Suite ${ }^{\mathrm{TM}}$ (IBM Academic Initiative Program)). As you know the description of an SDL system allows to specify the states machine of the communications protocol in a simple manner by means of a graphic interface in which the implied entities, the logical channels between them, the input/output signals, the states, etc. are specified The description of an SDL system is carried out at three levels: the system level, the block level and the process level. We are now going to present each of these three levels.

Two entities participate in the protocol that we have specified (FLSAP): the FLSA and the FLMSA. The description of the SDL system is described in Fig. 4. Two channels have been established $\mathrm{C} 1$ and $\mathrm{C} 2$, as have four signals (Access_Points, Location, Get_APs, MS_RSSI). The C3 channel and the "Get_Location" signal allow us to initiate the simulation of the behavior of the system.

FLSA entity initially sends the signal "Get_APs" to the FLSAP entity to obtain the Access Points that will be used to locate the MS. Then the FLSAP entity sends back to FLSA entity these Access Points with the "Access_Points" signal. At this time the FLSA entity collects the received signal strength (RSS) from the access points and sends this information to the FLSAP entity in the "MS_RSSI" signal. Finally, FLSAP entity sends back to FLSA entity the MS location on the floor with the "Location" signal.

The level of the "FLSA" block, described in Fig. 5 allows us to describe the input/output signals and channels of the FLSA entity. Note that this entity represents the location Agent Software installed in an MS. At the block level we have specified the "SCAN_RSS_APS" block that represents the process in charge of carrying out the gathering of the powers received from the access points of the floor that it will serve to the MS in order to determine its location. These Access Points have been communicated to the FLSA of the MS by means of an "Access_Points" signal. The "SCAN_RSS_APS" block receives the identity of the access points from the "MyAPs" signal and returns the power received from the access points in the "RSSI" signal. It must be noted that the parameters of these signals are structures defined at the system 


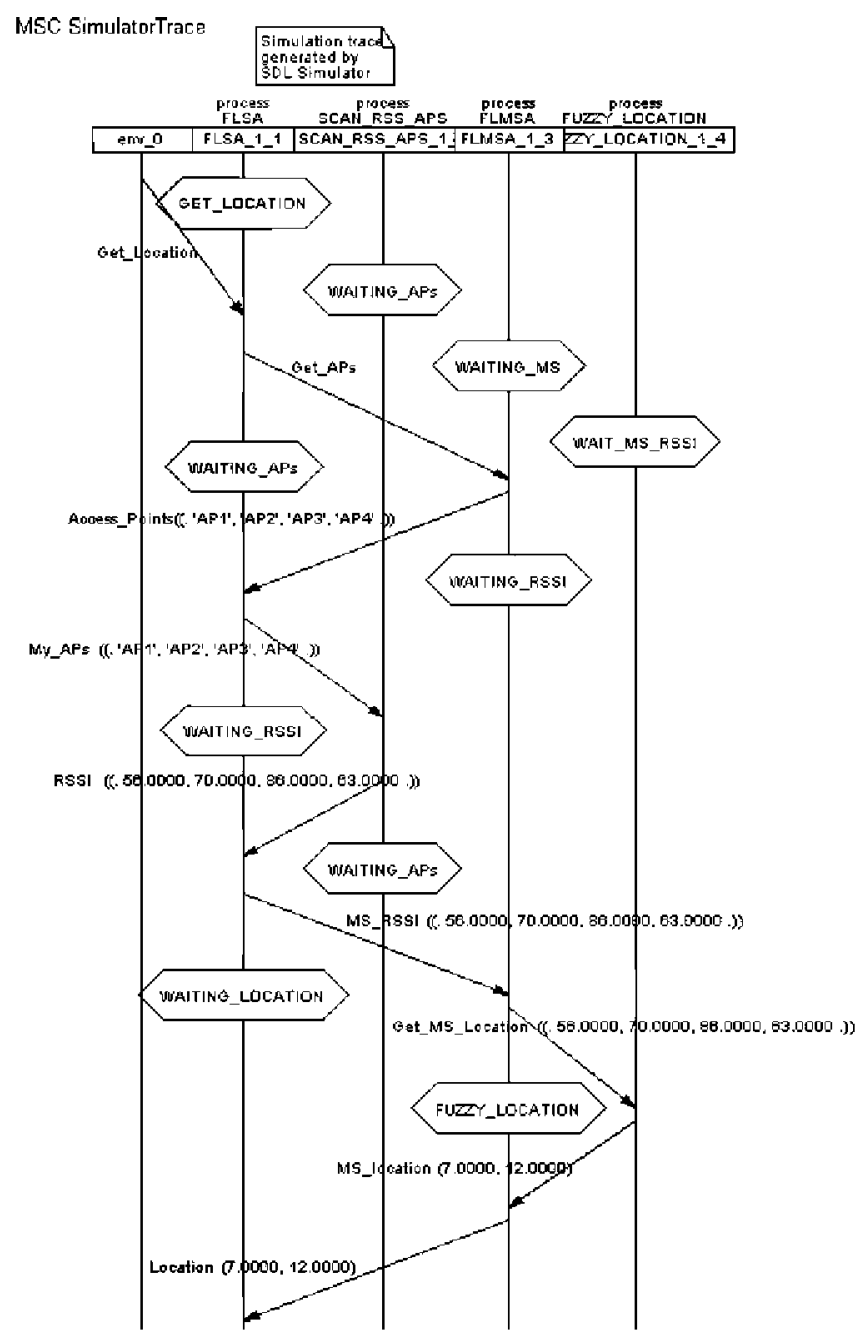

Fig. 8. MSC Diagram of the FLSAP protocol.

level. FLSA entity sends this information to the FLSMA entity in the "MS_RSSI" signal.

The "FLMSA", block level described in Fig. 6 allows us to discover the input/output signals and channels of the FLMSA entity. This entity represents the management agent software that will be in charge of locating the MS. At the block level we have specified the "FUZZY_LOCATION" block that represents the process in charge of locating the MS from the powers received at the Access points. These powers have been communicated to the FLSMA by means of the "MS_RSSI" signal.

The "FUZZY_LOCATION" block receives the power received by the MS from the Access points from the "Get_MS_location" signal and returns the user's location on the floor by means of the "MS_location" signal. The parameters of each of these signals are structured; defined at the system level. The internal working of the "FUZZY_LOCATION" process will be described later on and responds to a methodology based on the normalization of the measures received and the application of fuzzy rules for the establishment of the location on the floor. FLSMA entity sends the MS location to the FLSA entity in the "Location" signal.

The third level of the description of an SDL system is detailed in Fig. 7. This is the processing level. The finite state machine corresponding to FLSAP protocol is described.

The initial state of the FLSA is "Get_Location". This is the state in which the FLSA would be waiting for those high-level software entities required for their current location. This occurs when the
"Get_Location" signal arrives. From this moment on the FLSA requests the Access points from the FSMSA that are going to be used for location on the floor ("Get_APs" signal) and will remain waiting in the "WAITING_APs state". The FLMSA was initially in the "WAITING_MS" state.

Once the FLSA receives the identity of the APs ("Access_Points" signal), from the FLMSA it retransmits this information to the "SCAN_RSS_APS" process ("My_APs" signal) and waits to obtain the received RSSI power indicator of these APs ("WAITING_RSSI" state). Meanwhile the FLMSA is in the "WAITING_RSSI" state after sending the identity of the APs to the FLSA.

The "SCAN_RSS_APS" obtains the powers of the APs and sends them to the FSLA through the "RSSI" signal. The FSLA retransmits this information to the FLMSA through the "MS_RSSI" signal and goes into the "WAITING_LOCATION" state while waiting for the FLMSA to obtain the location of the MS. In the meantime the FLMSA goes from the "WAITING_RSSI" state to the "FUZZY_LOCATION" state after receiving the powers of the APs from the FLSA APs and having retransmitted this information to the "FUZZY_LOCATION" process.

The location of the MS on the plan of the floor by means of Fuzzy Controllers whose inputs are normalized measurements. The "FUZZY_LOCATION" process carries out this task by means of the "MS_Location" signal sending this information to the FLMSA. Finally, the FLSMA retransmits the MS by means of the "Location" signal.

\subsubsection{Behavior of the FLSAP protocol}

MSC (Message Sequence Charts) is a graphical and textual language for the description and specification of the interactions between the specific systems components e.g. with SDL. Message Sequence Charts may be used for requirement specification, simulation and validation, test-case specification and documentation of real-time systems.

In Fig. 8 we describe the behavior of the FLMSA protocol with MSC in which the dynamic system that we propose, through which the MS is located on the plan of the floor on which it is moved, is shown graphically.

\subsection{Fuzzy location methodology}

The location methodology based on fuzzy logic is going to permit the FLMSA to determine the location of an MS on the floor of the building. This location is a complement to the GPS location system so that we can follow the movement of an MS provided with 3G, 4G and Wi-Fi technology by means of the floors and rooms of a building.

The Location methodology that we are going to use consists of two phases. A WLAN location determination system usually works in two phases: an offline training phase (calibration phase) and online location determination phase. During the offline phase, the signal strength received from the access points, at selected locations in the area of interest, is tabulated resulting in a so-called radio-map. During the location determination phase, the signal strength samples received from the access points are used to "search" the radio map to estimate the user location.

However, in this paper we present a different way of considering the calibration phase and that of the determination of the location. In our system the power measurements received are normalized as regards locations hidden in the areas closest to the access points. In this way we can build not one but K Radio Maps, as hidden locations close to the APs, which allows us to place the mobile stations optimally.

The building of the Radio Maps is carried out by means of masks that represent the increase/decrease in the power received from 
the AP's in relation to the power received at the reference locations. (See Fig. 9).

We also normalize the current power received by MS (mobile station) in respect to " $K$ " locations in the estimation phase.

The measurements of the powers received at locations close to the APs follow a pattern independently of the manufacturer of the Wi-Fi cards. The power received from the closest AP is much greater than the rest of the powers received. The rest of the powers received from the other access points will depend on the obstacles, thickness and quality of the walls etc., but in any case there is a pattern of increase/decrease that complies with any Wi-Fi interface.

If, when an MS passes by an AP, the said AP is taken as a reference and with the power references of the Radio Map created for this access point we are able to obtain an estimation of the movement of the MS on the plan of the floor.

The proposed system requires a minimum processing cost and is independent of the manufacturer of the wireless interface since it is based on the increase/decrease in the powers received in relation to the reference locations.

\subsubsection{Calibration phase. Construction of $K$ Radio-Maps}

In order to explain the working of this phase, we are going to give an example of one in which we have experienced the results that we present in this paper (see Fig. 10). Fig. 10 gives an overview of the experimental environment. The figure shows the floor of part of our workplace. The total surface area ranges from 300 to $500 \mathrm{~m} 2$. Faculty members work in separate rooms. The walls are very thin (plaster). This is an obstacle especially for locating MSs in adjacent offices because of the reception of similar powers from the APs.
As a requirement for the working of our system, we assume that there $\mathrm{K}$ access points distributed around the floor being studied in order to locate an MS. Another requirement of our system is the establishment of $\mathrm{K}$ reference locations (close to the Access points) which will be a reference for measuring the powers.

In Fig. 10 we can see the floor being studied and on which the Access points AP1, AP2, AP3, and AP4 have been distributed symmetrically. Four reference locations: P1, P4, P6 and P7 have also been located, close to the AṔs and which serve to normalize the powers at any place on the floor.

In our system, the calibration phase consists of taking 100 samples ( 1 sample/s in every room (we are going to assume that there are $\mathrm{N}$ rooms). This is carried out with any wireless card and will be valid in the future to locate any MS independently of the wireless interface being used. The detailed algorithm is shown in Algorithm 1

We are going to identify a sample as a vector $m_{j}=\left(s_{1}, \ldots, s_{k}\right)$ which represents the measurement of power observed in place " $j$ " in the study. Where $s_{1}$ is the power observed from Access Point $1, s_{k}$ is the power observed from Access Point $K . .$.

A statistical average of the power samples observed is made from the samples taken, $m$ being the number of samples taken.

$M_{j}=\left(\frac{\sum_{l=1}^{m} s_{1_{l}}^{j}}{m}, \frac{\sum_{l=1}^{m} s_{2_{l}}^{j}}{m}, \ldots, \frac{\sum_{l=1}^{m} S_{K_{l}}^{j}}{m}\right)=\left(S_{1}^{j}, S_{2}^{j}, \ldots, S_{K}^{j}\right)$

The vector $M_{j}=\left(S_{1}{ }^{j}, \ldots, S_{K}{ }^{j}\right)$, is finally the representation of the power received at place " $\mathrm{j}$ ".

We propose that each power vector $M_{j}$ obtained is expanded to $K$ vectors, the result of normalizing the measured powers as regards the $K$ reference locations ( $k$ being equal to the number of access

Algorithm 1.Construction of K Radio-Maps

Alg.1 $\left[\left(\operatorname{mask}_{1_{j}}^{P_{i}}, \operatorname{mask}_{2_{j}}^{P_{i}}, \ldots, \operatorname{mask}_{K_{j}}^{P_{i}}\right)_{j=1, \ldots, N}\right]=\operatorname{Get}$ RadioMap $(m$, ReferenceLocations $)(N=$ number of locations $)$,

$(i=1, \ldots, K), K=$ number of access points

Require

$M_{j}$ : Measure Signal Strength Vector of a MS in location $j$ from $K$ access points $\left(M_{j}=\left(S_{1}^{j}, \ldots, S_{K}^{j}\right)\right)$

Set Referencelocations: $\left(\mathrm{P}_{1}, \ldots, \mathrm{P}_{\mathrm{K}}\right)$

$\left.M_{(} p_{j}\right)$ : Measure Signal Strength Vector of a MS in the reference locations $\left\{M_{P_{i}}=\left(S_{1}^{P_{i}}, \ldots, S_{K}^{P_{i}}\right)\right\}(i=1, \ldots, K)$

Ensure

Mask of Measured Signal Strength increase/decrease from reference locations

$1: M_{j},\left[M_{j}=\left(S_{1}^{j}, \ldots, S_{K}^{j}\right)\right]_{j=1, \ldots, N} \quad$ Get Current RSSI of MS(For all locations in the floor)

2: $M_{P_{i}}=\left(S_{1}^{P_{i}}, \ldots, S_{K}^{P_{i}}\right) \quad$ Get Signal Strength of Locations Refrence $(i=1, \ldots, K)$

4: for $(i=1 ; i=K ; i++) M_{j}$, generamos $\rightarrow n M_{j}^{P_{i}}=\left[\left(S_{1}^{P_{i}}-S_{1}^{j}\right), \ldots,\left(S_{K}^{P_{i}}-S_{K}^{j}\right)\right]=\left[\Delta S_{1}^{P_{i, j}}, \ldots, \Delta S_{K}^{P_{i j}}\right](j=1, \ldots N)$

\{Normalized measures from a location $P_{i}$, Measured Signal Strength increase/decrease from a reference location $\}(i=1, \ldots, K)$

5: $n M_{j}^{P_{i}}(j=1, \ldots, N)(i=1, \ldots, K)$

If $\left|\Delta S_{l}^{P_{i, j}}\right|<10 \mathrm{~dB} \rightarrow \operatorname{mask}_{l j}^{P_{i}}=" * "(l=1, \ldots, K)$

else if $10 \mathrm{db}<\left|\Delta S_{l}^{P_{i, j}}\right|<20 \mathrm{~dB} \rightarrow \operatorname{mask}_{l j}^{P_{i}}="+/-" 1$

else if $\left|\Delta S_{l}^{P_{i, j}}\right|>20 \mathrm{~dB} \leftarrow \operatorname{mask}_{l j}^{P_{i}}="+/-2 "$

$\left[\left(\operatorname{mask}_{1_{j}}^{P_{i}}, \operatorname{mask}_{2_{j}}^{P_{i}}, \ldots, \operatorname{mask}_{K_{j}}^{P_{i}}\right)_{j=1, \ldots, N}\right]=\leftarrow n M_{j}^{P_{i}}(j=1, \ldots, N)(i=1, \ldots, K)$

\{All normalized measures are converted into masks\} 


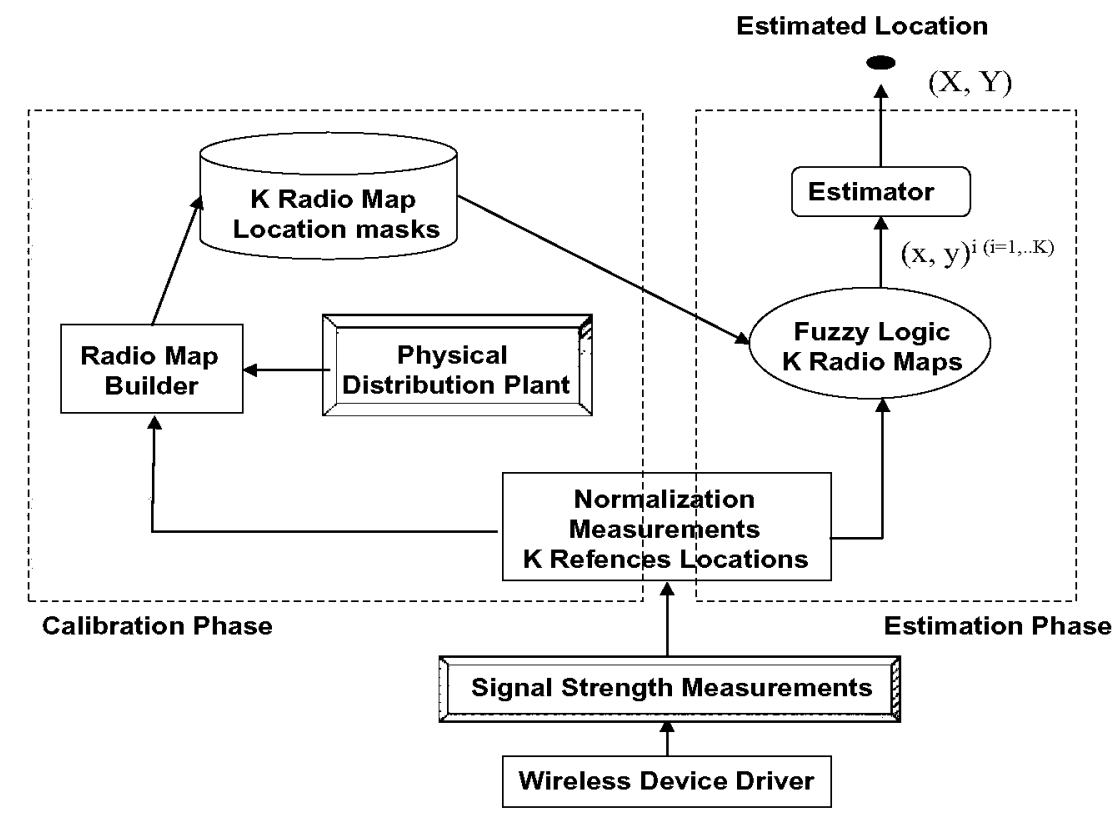

Fig. 9. Location estimation methodology structure.

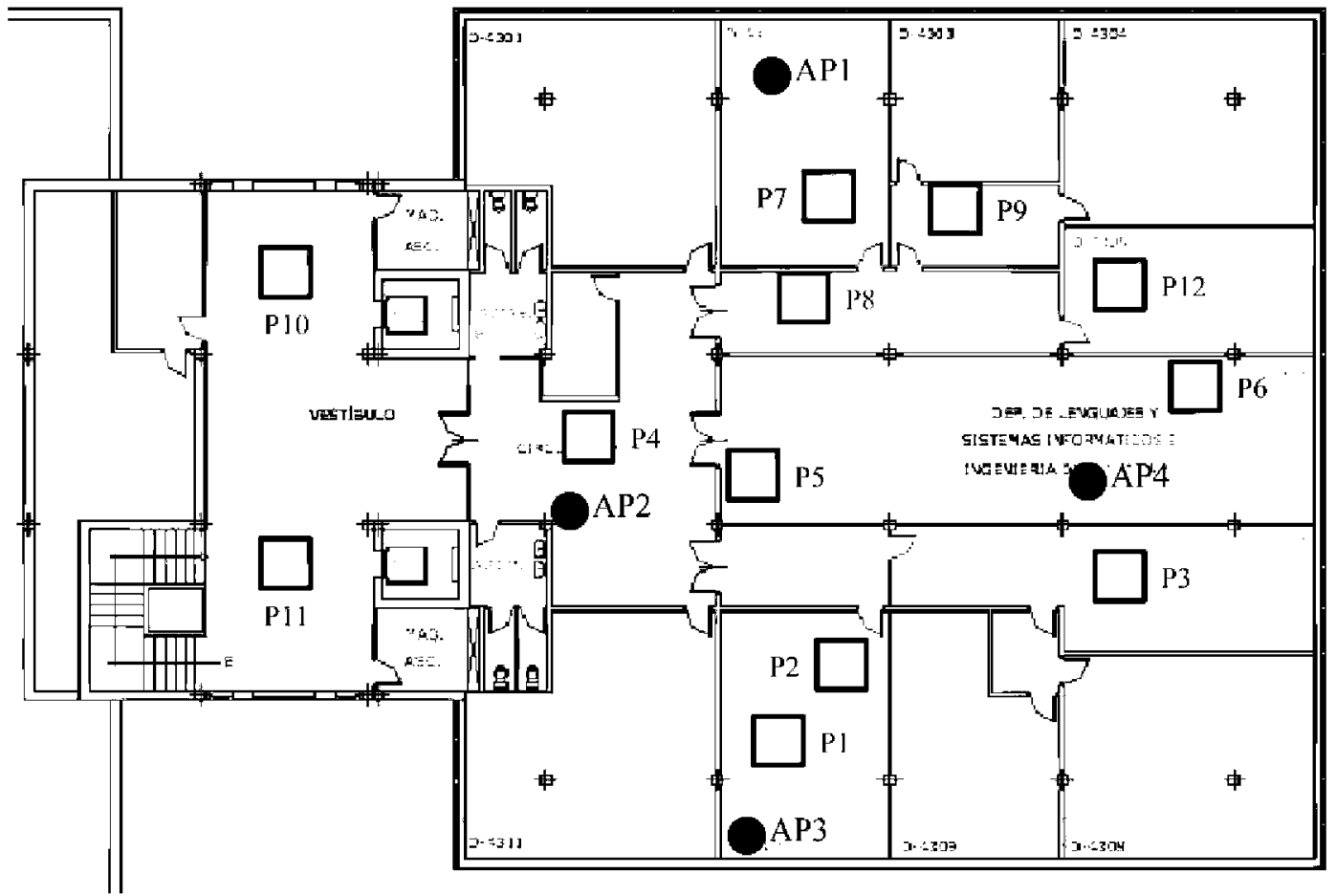

Fig. 10. Floor plan of our workplace.

points). That is, we have a new vector for each reference location for the room $j$. Every one of these vectors represents the increase/decrease in power in relation to the reference locations. All of this procedure will be applied to the $N$ rooms on the floor being studied.

$n M_{j}^{P i}=\left[\left(S_{1}^{P i}-S_{1}^{j}\right), \ldots,\left(S_{k}^{P i}-S_{k}^{j}\right)\right]=\left[\Delta S_{1}^{P i, j}, \ldots, \Delta S_{k}^{P i, j}\right]$

$(j=1, \ldots, N)(i=1, \ldots, K)$

Once we have identified the place " $j$ " by " $K$ " normalized vectors, we construct the $K$ reference Radio Maps that will serve to locate any MS in the position estimation phase.
In order to do this we transform the normalized vectors of each location " $j$ " into mask vectors. A mask is the representation of the increase/decrease in power as regards the reference locations.

The masks that we use in our system have the following values:

- The value "**" means that there is no significant increase in measured power in respect to a reference position (values of less than $10 \mathrm{~dB}$ )

- The value " $+1 /-1$ " means that there is a moderate variation (either increment or decrement respectively) with respect to the reference position that oscillates between 10 and $20 \mathrm{~dB}$. 


\section{Normalization Reference P1 $\quad[\mathbf{A P} 1, \mathbf{A P} 2, \mathbf{A P 3}, \mathbf{A P} 4]$}

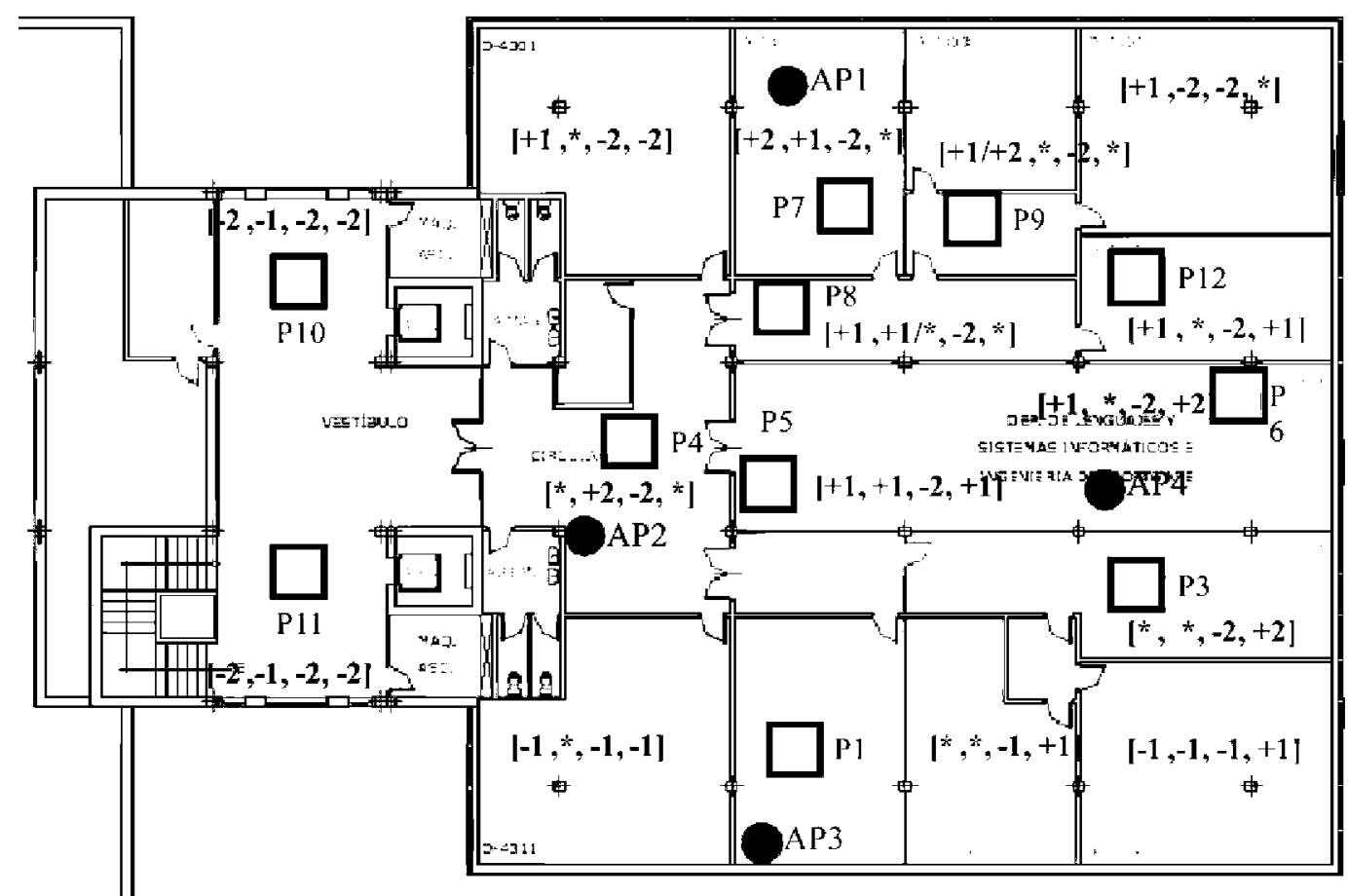

Fig. 11. Normalized measurements from location P1.

- The value " $+/-2$ " means that there is a large variation (either increment or decrement respectively) with respect to the reference position that oscillates between values more than $20 \mathrm{~dB}$.

In Fig. 11 the Radio Map constructed by taking place P1 as reference can be seen (see Fig. 13-16). ing. These powers are received and evaluated by the FLSMA with the aim of estimating the location. It is during this phase of the estimation methodology that the position of the MS on the floor of the building is established.

A description of the algorithm used to evaluate the estimation of the location is presented in Algorithm 2:

\section{Algorithm 2. MS position estimation.}

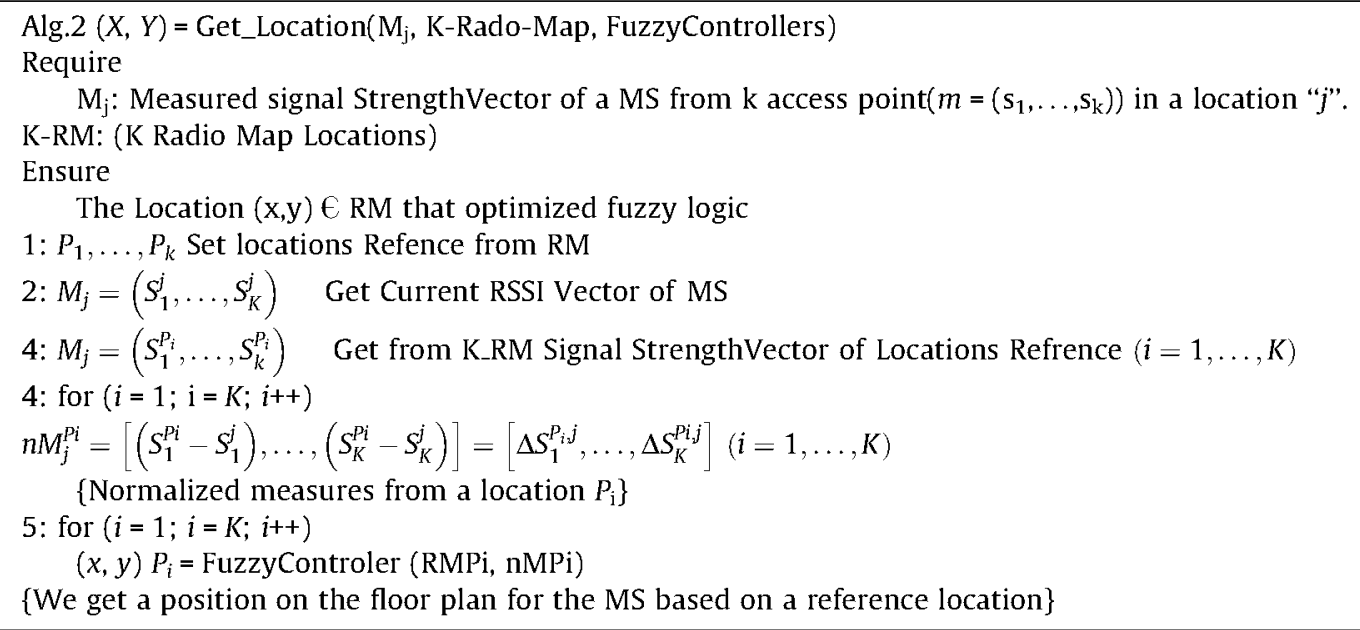

3.3.2. Location estimation phase. Description of the fuzzy logic system In the Location Estimation Phase, the FLSA of the MS gathers the powers received from the APs distributed on the floor of the build-
An MS is any wireless interface that moves around the floor being studied receiving a Beacon connection from the surrounding access points. At a given moment this MS obtains a power value re- 
ceived from the surrounding $K$ APs equal to a vector $m=\left(s_{1}, \ldots, s_{k}\right)$, where $s_{1}$ is the power observed from Access Point $1, s_{k}$ is the power observed from Access Point $K$...

After gathering $\mathrm{m}$ samples in the current position a vector of powers $M_{j}=\left(S_{1}^{j}, \ldots, S_{K}^{j}\right)$ is constructed.

$M_{j}=\left(\frac{\sum_{l=1}^{m} s_{1_{1}}^{j}}{m}, \frac{\sum_{l=1}^{m} s_{2_{l}}^{j}}{m}, \ldots, \frac{\sum_{l=1}^{m} S_{k_{l}}^{j}}{m}\right)=\left(S_{1}^{j}, S_{2}^{j}, \ldots, S_{k}^{j}\right)$

The vector $M_{j}$ is the representation of the power received at the current place " $j$ ". The obtained power vector $M_{j}$ is expanded to $K$ vectors as a result of normalizing the measured powers as opposed to $K$ reference locations (equal to the number of access points). All of this in a similar way to the construction of the $K$ Radio Maps

$n M_{j}^{P i}=\left[\left(S_{1}^{P i}-S_{1}^{j}\right), \ldots,\left(S_{k}^{P_{i}}-S_{k}^{j}\right)\right]=\left[\Delta S_{1}^{P_{i j}}, \ldots, \Delta S_{k}^{P_{i j} j}\right]$

$(i=1, \ldots, K)$

For this reference plan under consideration and with each normalized vector obtained in this plan, we apply a Fuzzy Controller which will give us an approximation of the location $(x, y)^{\mathrm{Pi}}$ on this estimation plan.

Our location estimation methodology is going to determine which radio-map to use in accordance with the MS. When an MS moves within the proximity of any reference location, the reference radio-map of this location will be taken in order to evaluate the position of the MS.

The variables to be considered for locating mobile stations are the received signal strength indicator (RSSI) measurements from the $\mathrm{n}$ Access Points. However these measurements will be normalized with respect to the reference value locations near the different access points.

This means that we have n Fuzzy Controllers. The first one works with the RSSI measurements normalized with respect to the reference value locations near Access Point 1. The Second Fuzzy Controller works with the RSSI measurements normalized with respect to the reference value locations near Access Point 2 and so on.

The Fig. 12 shows the implementation of the Fuzzy Controller with the values normalized with respect to the location of the AP1.

As regards the Normalized Measurements (see Fig. 13) we define the following membership functions with linguistic variables/labels: Low $(-10,10 \mathrm{~dB})$, Medium-Low $(-20,-5 \mathrm{~dB})$, VeryLow (-15 dB,-), Medium-High ( $5,20 \mathrm{~dB}$ ), Very-High (15 dB-). We consider that a Low value means that the power received by the MS does not vary the power received at the reference place (in the figure, the place close to AP1). A Very-High or Very-Low value means that there has been a significant increase in the average power. Finally, Medium-High or Medium-Low means that there has been a slight increase/decrease in the average power.

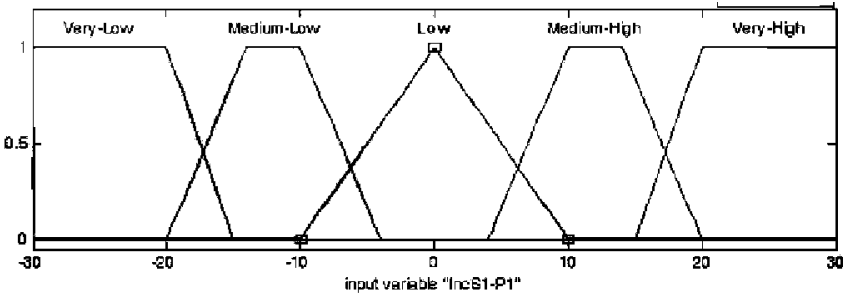

Fig. 13. Membership functions for fuzzy set of InS1-P1.

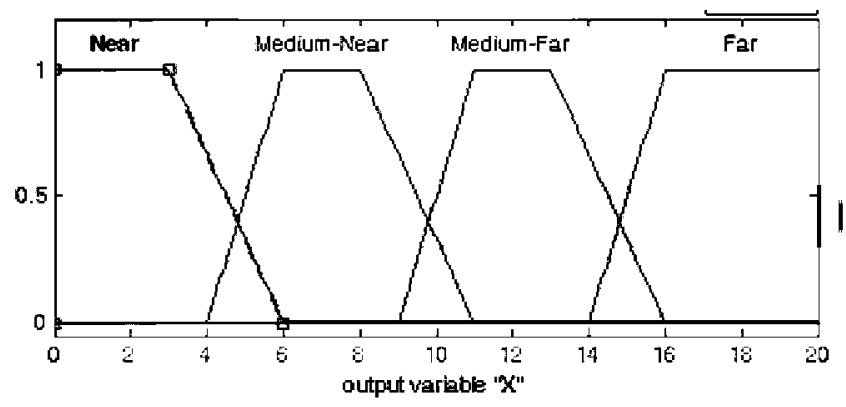

Fig. 14. Membership functions for fuzzy set output $X$.

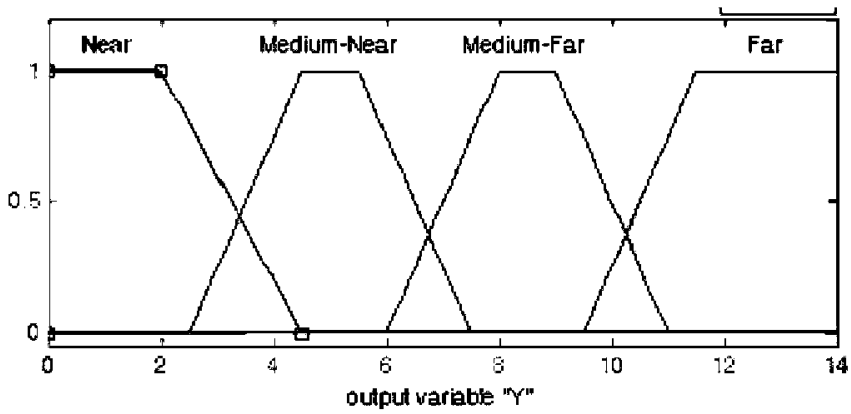

Fig. 15. Membership functions for fuzzy set output $Y$.

Bear in mind that the normalized measurements $(\mathrm{Nm})$ represents an increase/decrease in the measured power in relation to the measured power in the locations close to the access point

We finally define the following membership functions for the $X$ and $Y$ coordinates (see Figs. 14 and 15), that we will provide the estimation of the position on the plan being studied. The linguistic variables/labels are in the case of the $X$ abscissa: Near (0-6), Med-

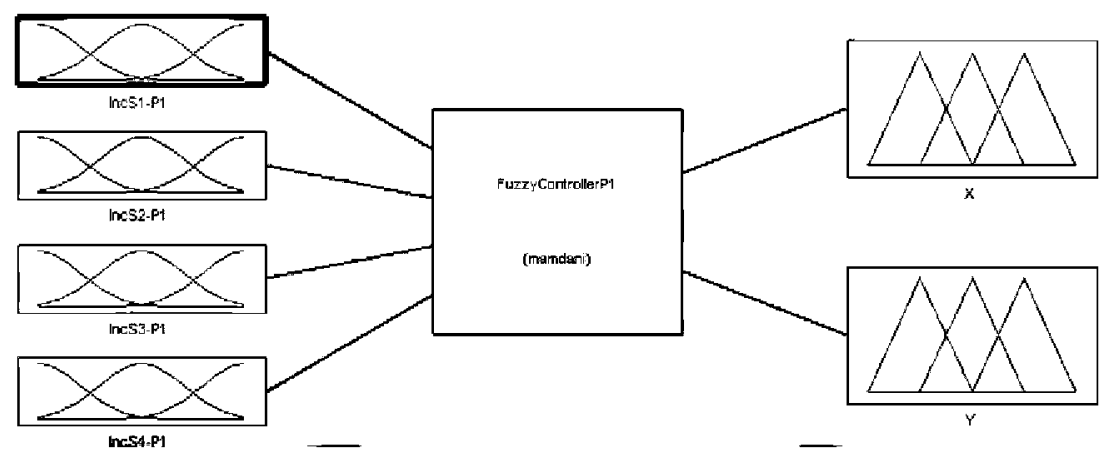

Fig. 12. Fuzzy controller schema. 


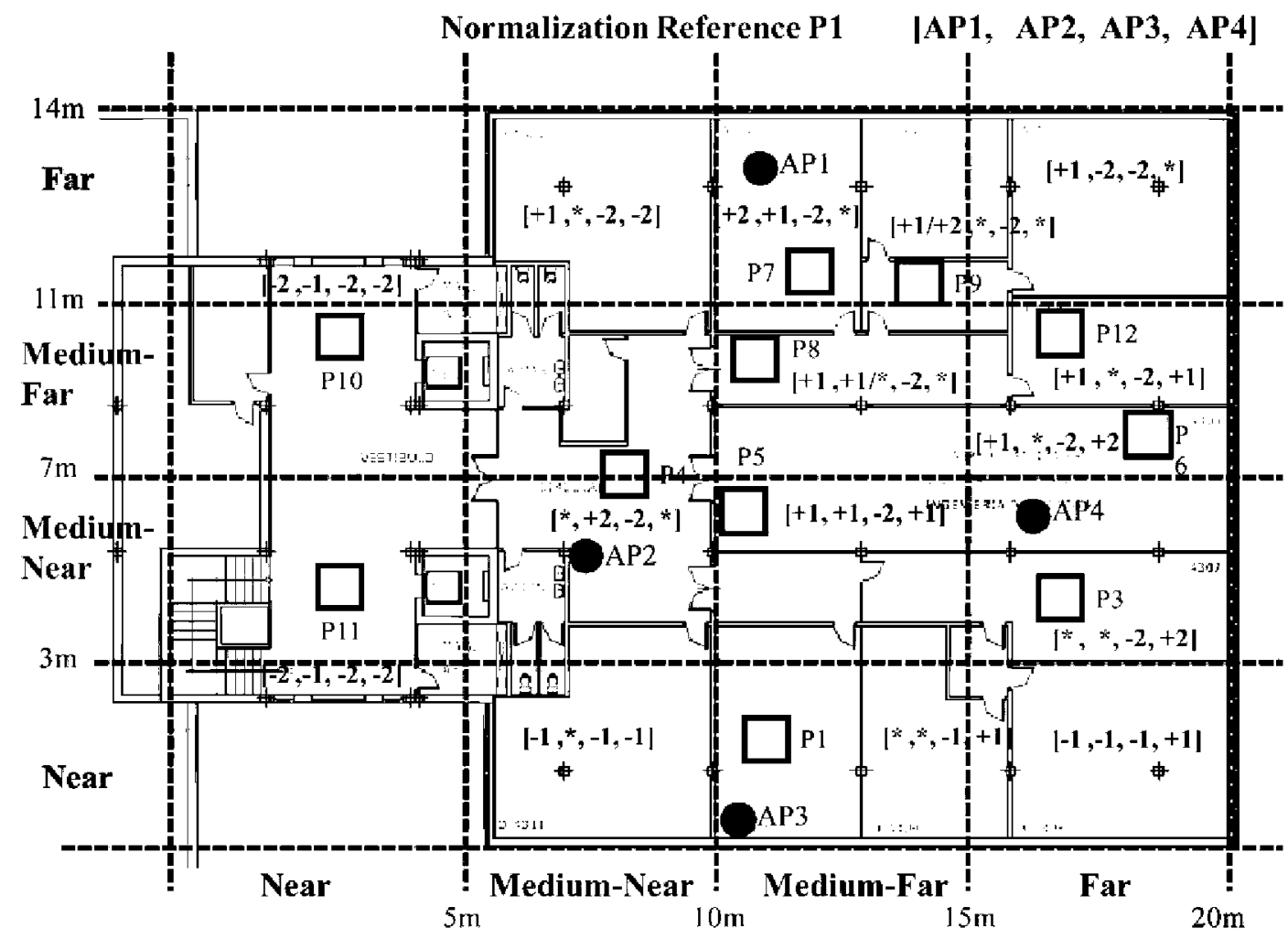

Fig. 16. Plan of the floor used by the Fuzzy Controllers.

ium-Near (4-11), Medium-Far (9-16), Far (14-). In the case of the Y ordinate the linguistic variables/labels are: Near (0-5), MediumNear (3-7), Medium-Far (6-11), Far (9-).

The position (coordinates $X$ and $Y$ ) on the floor of the building being studied is set out in the Fig. 16:

The rules of the fuzzy group, which are the knowledge base, are described in Table 1. In this table, 12 fuzzy rules were defined to govern the controllers' decisions.

\section{Results of evaluation experiments}

We applied our location-estimation methodology shown in Section 4 to implement an estimation location system and conducted evaluation experiments in our workplace. Fig. 10 gives an overview of the experimental environment.

Red circles show the location of each AP that we used in our tests. Green squares show the locations (12) at which we took measure- ments of the four AP signal strengths. The normalization points are $\mathrm{P} 1, \mathrm{P} 4, \mathrm{P} 6$ and $\mathrm{P7}$. In the survey step, we observed signal strength for 4 minutes and took 240 measurements at each location.

The hardware that we used in our experiments included four different IEEE 802.11 wireless adapters; 3Com, SMC, Ovislink and US Robotics. APs are manufactured by $3 \mathrm{Com}$ and US Robotics

As the first step in the explanation of our method, we are going to discuss an example using the experimental values (absolute or pre-normalized values) obtained by different cards in the same place (any place) on the floor in question:

$$
\begin{aligned}
& m_{i, 3 \mathrm{com}}=(-92,-74,-67,-87) \\
& m_{i, \text { Ovislink }}=(-75,-54,-49,-64) \\
& m_{i, \text { SMC }}=(-82,-71,-53,-79) \\
& m_{i, \text { USRobotics }}=(+56,+70,+86,+63)
\end{aligned}
$$

Note that, as mentioned in Section 3, there is a wide range of values This makes it impossible to apply Fuzzy Controllers directly. How-

Table 1

Fuzzy rules radio-map reference location $\mathrm{P} 1$.

1. If (lncS1P1 is Low) and (lncS2P1 is Low) and (lncS3P1 is Low) and (lncS4P1 is Low) then ( $x$ is Medium-Far) ( $y$ is Near)

2. If (lncS1P1 is Low) and (lncS2P1 is Very-High) and (lncS3P1 is Very-Low) and (lncS4P1 is Low) then ( $x$ is Medium-Near) ( $y$ is Medium-Near)

3. If (lncS1P1 is Medium-High) and (lncS2P1 is Medium-High) and (lncS3P1 is Very-Low) and (lncS4P1 is Medium-High) then ( $x$ is Medium-Far) ( $y$ is Medium-Near)

4. If (lncS1P1 is Medium-High) and (lncS2P1 is Medium-High) and (lncS3P1 is Very-Low) and (lncS4P1 is Low) then ( $x$ is Medium-Far)( $y$ is Medium-Far)

5. If (lncS1P1 is Medium-High) and (IncS2P1 is Low)and(lncS3P1 is Very-Low) and (lncS4P1 is Low) then ( $x$ is Medium-Far)( $y$ is Medium-Far)

6. If (lncS1P1 is Very-High) and (lncS2P1 is Medium-High) and (lncS3P1 is Very-Low) and (lncS4P1 is Low) then ( $x$ is Medium-Far) ( $y$ is Far)

7. If (1ncS1P1 is Medium-High) and (nncS2P1 is Low) and (lncS3P1 is Very-Low) and (1ncS4P1 is Low) then ( $x$ is Medium-Far) ( $y$ is Far)

8. If (1ncS1P1 is Very-High) and (ncS2P1 is Low) and (1ncS3P1 is Very-Low) and (1ncS4P1 is Low) then ( $x$ is Medium-Far) ( $y$ is Far)

9. If (1ncS1P1 is Medium-High) and (lncS2P1 is Low) and (1ncS3P1 is Very-Low) and (1ncS4P1 is Medium-High) then ( $x$ is Far) ( $y$ is Medium-Far)

10. If (IncS1P1 is Medium-High) and (lncS2P1 is Low) and (lncS3P1 is Very-Low) and (lncS4P1 is Very-High) then ( $x$ is Far) ( $y$ is Medium-Far)

11. If (IncS1P1 isLow) and (lncS2P1 is Low) and (IncS3P1 is Very-Low) and (lncS4P1 is Very-High) then ( $x$ is Far) ( $y$ is Medium-Near)

12. If (IncS1P1 is Medium-High) and (lncS2P1 is Low) and (lncS3P1 is Very-Low) and (lncS4P1 is Very-Low) then ( $x$ is Medium-Near) ( $y$ is Far)

13. If (IncS1P1 is Medium-High) and (lncS2P1 is Very-Low) and (lncS3P1 is Very-Low) and (lncS4P1 is Lowjthen ( $x$ is Far) ( $y$ is Far)

14. If (lncS1P1 is Medium-Low) and (lncS2P1 is Medium-Low) and (lncS3P1 is Medium-Low) and (lncS4P1 is Medium-High) then ( $x$ is Far) ( $y$ is Near)

15.If (lncS1P1 is Medium-Low) and (lncS2P1 is Low) and (lncS3P1 is Medium-Low) and (lncS4P1 is Medium-Low) then ( $x$ is Medium-Near) ( $y$ is Near)

16. If (lncS1P1 is Low) and (lncS2P1 is Low) and (lncS3P1 is Medium-Low) and (lncS4P1 is Medium-High) then ( $x$ is Medium-Far) ( $y$ is Near) 


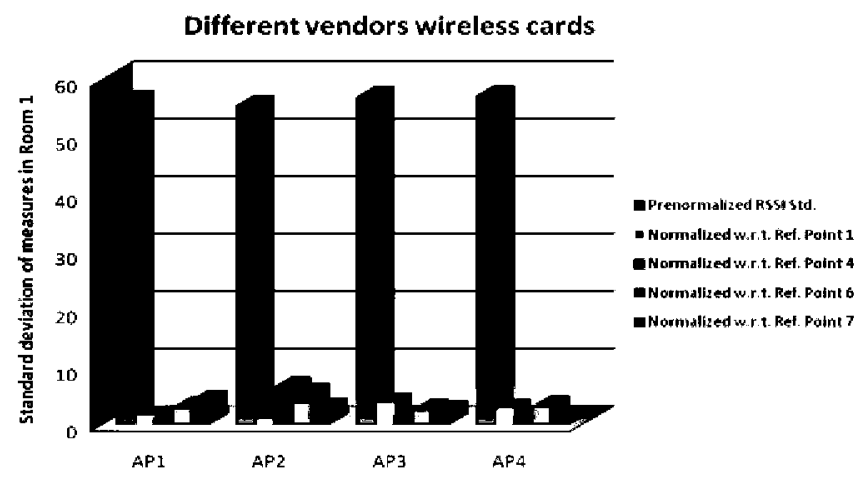

Fig. 17. Comparison of absolute/normalized measurement STD.

ever, we can transform these absolute values into relative values as explained in Section 3.3.1. This way, by normalizing the values with respect to the reference value $\mathrm{P} 1$ in each case, we get the expansion vectors:

$$
\begin{aligned}
& e_{i, 3 \text { com }}^{\mathrm{P} 1}=(-0.75,-0.02,-6.21,-2.69) \\
& e_{i, \text { Ovislink }}^{\mathrm{P} 1}=(+1.83,-0.11,-7.13,+4.72) \\
& e_{i, \mathrm{SMC}}^{\mathrm{P} 1}=(-0.29,+0.67,+2.27,+1.99) \\
& e_{i, \text { USRobotics }}^{\mathrm{P}}=(+0.63,-1.48,+0.94,-0.78)
\end{aligned}
$$

By normalizing the values with respect to the reference point $P 4$, we get:

$e_{i, 3 \mathrm{com}}^{\mathrm{P} 4}=(-5.52,-21.87,+19.63,+0.12)$

$e_{i, \text { Ovislink }}^{\mathrm{P} 4}=(-6.46,-24.61,+19.49,+3.04)$

$e_{i, \mathrm{SMC}}^{\mathrm{P} 4}=(-7.07,-31.10,+22.51,-0.01)$

$e_{i, \mathrm{USR} \text { obotics }}^{\mathrm{P} 4}=(-8.18,-34.09,+22.76,+1.58)$
By normalizing the values with respect to the reference point P6, we get:

$$
\begin{aligned}
& e_{i, 3 \text { com }}^{\mathrm{PG}}=(-7.34,+0.83,+22.18,-28,67) \\
& e_{i, \text { Ovislink }}^{\mathrm{PG}}=(-13.61,+1.34,+23.74,-19.89) \\
& e_{i, \mathrm{SMC}}^{\mathrm{P} 6}=(-12.67,-5.43,+26.58,-25.12) \\
& e_{i, \mathrm{USR} \text { obotics }}^{\mathrm{PG}}=(-11.83,-7.51,+29.24,+22.24)
\end{aligned}
$$

and, finally, by normalizing the values with respect to the reference value P7, we get:

$$
\begin{aligned}
& e_{i, 3 \text { com }}^{\mathrm{P7}}=(-23.92,+6.12,+35.59,+2.01) \\
& e_{i, \text { Ovislink }}^{\mathrm{P}}=(-35.82,+9.74,+34.58,+3.03) \\
& e_{i, \text { SMC }}^{\mathrm{P7}}=(-31.08,+4.74,+37.37,+1.78) \\
& e_{i, \text { USRobotics }}^{\mathrm{P7}}=(-33.73,+2.09,+34.87,+2.24)
\end{aligned}
$$

After analyzing the experimental results, we find that we have managed to get very similar relative, card-independent values using this method. This is because we measure the relative difference between two points across all the cards. This way, we can enter these new relative data into our fuzzy system. The deviations in the measurements, as shown in Fig. 17, are insignificant compared with the absolute values of the analyzed cards.

\subsection{Location of an MS at locations close to the access points}

The statistical analysis of the power measurements received from the access points allows us to establish easily the position of a mobile device at locations close to the access points. At these levels the level of the power received from the access point is much higher than the rest of the APs and the power received from the rest of the access points has a pattern derived from the characteristics of the plan being studied. For example, we present the pow-

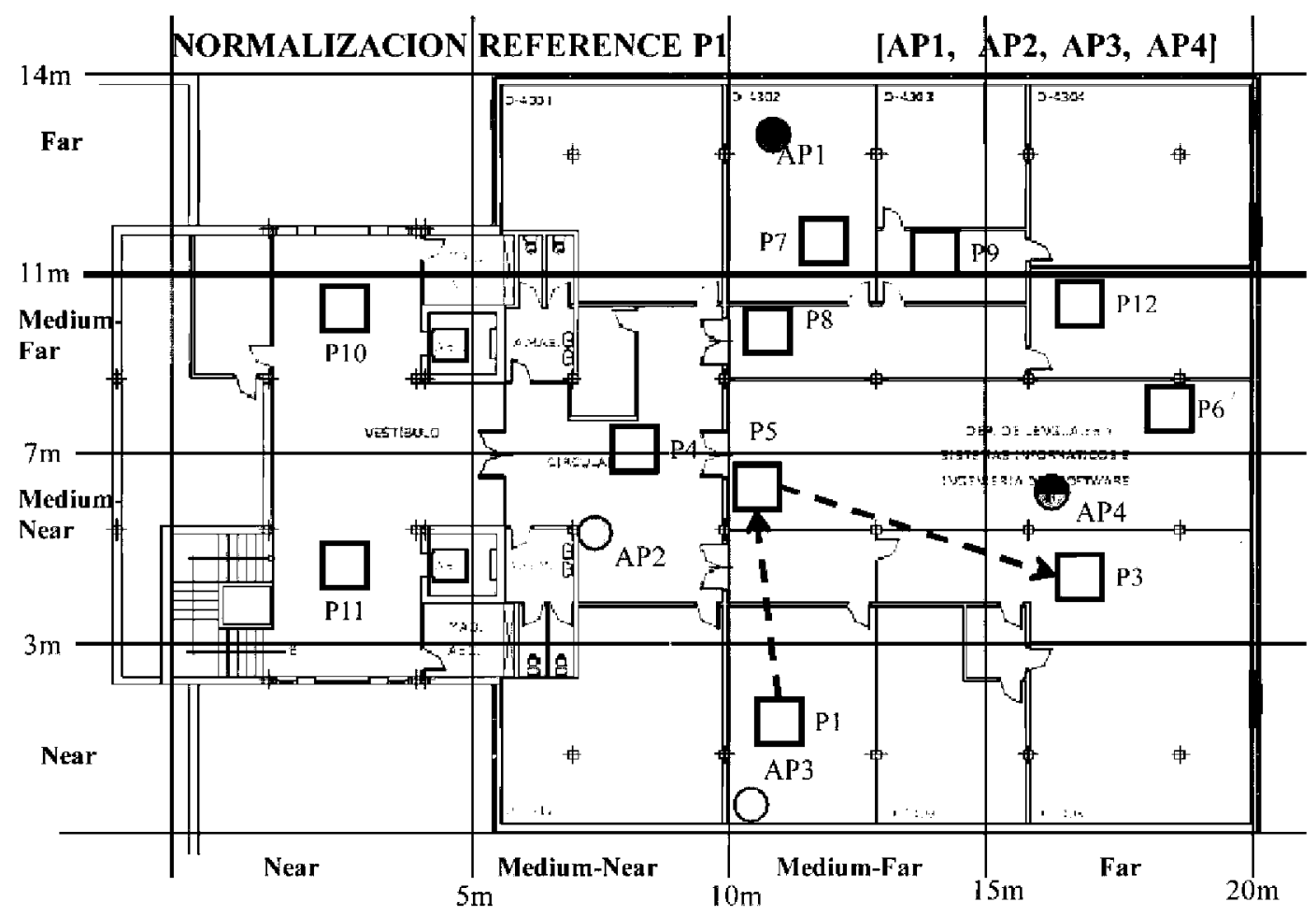

Fig. 18. MS Route, Experiment 1. 
Table 2a

Received signal strength (RSS) location P1, 3Com.

\begin{tabular}{|c|c|c|c|}
\hline $\begin{array}{l}\text { 3com Wireless card } \\
\text { APMAC }\end{array}$ & RSSI & Mean & Standard deviation \\
\hline AP2 (00-c0-49-f2-38-92) & -73.98 & 3.33 & {$[-70.644516,-77.311035]$} \\
\hline AP1 (00-14-c 1-03-96-46) & -91.25 & 1.17 & {$[-90.086110,-92.419642]$} \\
\hline AP3(00-04-76-a7-b1-bf) & -60.79 & 4.94 & {$[-55.845891,-65.731883]$} \\
\hline AP4 (00-0a-e9-0a-0b-5f) & -84.31 & 2.26 & {$[-82.052082,-86.570141]$} \\
\hline \multirow{2}{*}{\multicolumn{4}{|c|}{$\begin{array}{l}\text { AP of greatest RSSI is AP3 (00-04-76-a7-b1-bf) } \\
\text { Normalizing with respect AP of greatest RSSI }\end{array}$}} \\
\hline & & & \\
\hline APMAC & Mean & Standard deviation & \\
\hline AP2 (00-c0-49-f2-38-92) & 13.19 & {$[16.522148,9.855629]$} & \\
\hline AP1 $(00-14-c 1-03-96-46)$ & 30.46 & [31.630755, 29.297223] & \\
\hline AP3(00-04-76-a7-b1-bf) & 0.00 & {$[4.942996,-4.942996]$} & \\
\hline AP4 (00-0a-e9-0a-0b-5f) & 23.52 & [25.781254, 21.263195] & \\
\hline
\end{tabular}

Table 2b

Received signal strength (RSS) location P1, SMC.

\begin{tabular}{|c|c|c|c|}
\hline $\begin{array}{l}\text { SMC wireless card } \\
\text { APMAC }\end{array}$ & RSSI & Mean & Standard deviation \\
\hline AP2 (00-c0-49-f2-38-92) & -71.67 & 3.44 & {$[-68.221863,-75.111466]$} \\
\hline AP4 (00-0a-e9-0a-0b-5f) & -80.99 & 3.43 & {$[-77.558652,-84.419131]$} \\
\hline AP3(00-04-76-a7-b1-bf) & -55.27 & 3.19 & {$[-52.080587,-58.452746]$} \\
\hline AP1 $(00-14-c 1-03-96-46)$ & -81.71 & 2.58 & {$[-79.132376,-84.289850]$} \\
\hline \multirow{2}{*}{\multicolumn{4}{|c|}{$\begin{array}{l}\text { AP of greatest RSSI is AP3 (00-04-76-a7-b1-bf) } \\
\text { Normalizing with respect AP of greatest RSSI }\end{array}$}} \\
\hline & & & \\
\hline APMAC & Mean & Standard deviation & \\
\hline AP2 (00-c0-49-f2-38-92) & 16.40 & {$[19.844799,12.955196]$} & \\
\hline AP4 (00-0a-e9-0a-0b-5f) & 25.72 & [29.152464, 22.291986] & \\
\hline AP3(00-04-76-a7-b1-bf) & 0.00 & {$[3.186080,-3.186080]$} & \\
\hline AP1 $(00-14-\mathrm{C} 1-03-96-46)$ & 26.44 & {$[29.023184,23.865710]$} & \\
\hline
\end{tabular}

Table 2c

Received signal strength (RSS) location P1, Ovislink.

\begin{tabular}{|c|c|c|c|}
\hline $\begin{array}{l}\text { Ovishnk wireless Card } \\
\text { APMAC }\end{array}$ & RSSI & Mean & Standard deviation \\
\hline $\mathrm{AP} 2(00-\mathrm{C} 0-49-\mathrm{f} 2-38-92)$ & -53.89 & 1.97 & {$[-51.919995,-55.857783]$} \\
\hline AP1 (00-14-c1-03-96-46) & -76.83 & 3.29 & {$[-73.542777,-80.123895]$} \\
\hline AP3(00-04-76-a7-b1-bf) & -41.87 & 2.78 & {$[-39.084979,-44.648351]$} \\
\hline AP4 (00-0a-e9-0a-0b-5f) & -68.72 & 3.31 & {$[-65.408855,-72.035587]$} \\
\hline \multirow{2}{*}{\multicolumn{4}{|c|}{$\begin{array}{l}\text { AP of greatest RSSI is AP3 (00-04-76-a7-b1-bf) } \\
\text { Normalizing with respect AP of greatest RSSI }\end{array}$}} \\
\hline & & & \\
\hline APMAC & Mean & Standard deviation & \\
\hline $\mathrm{AP} 2(00-\mathrm{c} 0-49-\mathrm{f} 2-38-92)$ & 12.02 & {$[13.991118,10.053331]$} & \\
\hline AP1 (00-14-c1-03-96-46) & 34.97 & {$[38.257230,31.676112]$} & \\
\hline $\mathrm{AP} 3(00-04-76-a 7-b 1-b f)$ & 0.00 & {$[2.781686,-2.781686]$} & \\
\hline AP4 (00-0a-e9-0a-0b-5f) & 26.86 & [30.168922, 23.542191] & \\
\hline
\end{tabular}

Table 2d

Received signal strength (RSS) location P1, Robotics.

\begin{tabular}{|c|c|c|c|}
\hline $\begin{array}{l}\text { Robotics wireless card } \\
\text { APMAC }\end{array}$ & RSSI & Mean & Standard deviation \\
\hline AP2 (00-c0-49-f 2-38-92) & 71.48 & 1.91 & {$[73.388144,69.567408]$} \\
\hline AP3(00-04-76-a7-b1-bf) & 85.06 & 0.99 & {$[86.048433,84.062681]$} \\
\hline AP4 (00-0a-e9-0a-0b-5f) & 63.78 & 1.88 & {$[65.662086,61.893471]$} \\
\hline AP1 (00-14-c 1-03-96-46) & 55.37 & 0.95 & {$[56.314762,54.418568]$} \\
\hline \multirow{2}{*}{\multicolumn{4}{|c|}{$\begin{array}{l}\text { AP of greatest RSSI is AP3 (00-04-76-a7-b1-bf) } \\
\text { Normalizing with respect AP of greatest RSSI }\end{array}$}} \\
\hline & & & \\
\hline APMAC & Mean & Standard deviation & \\
\hline AP2 (00-c0-49-f $2-38-92)$ & 13.58 & {$[15.488150,11.667414]$} & \\
\hline $\mathrm{AP} 3(00-04-76-a 7-b 1-b f)$ & 0.00 & {$[0.992876,-0.992876]$} & \\
\hline AP4 (00-0a-e9-0a-0b-5f) & 21.28 & {$[23.162086,19.393471]$} & \\
\hline AP1 (00-14-c 1-03-96-46) & 29.69 & {$[30.636990,28.740795]$} & \\
\hline
\end{tabular}




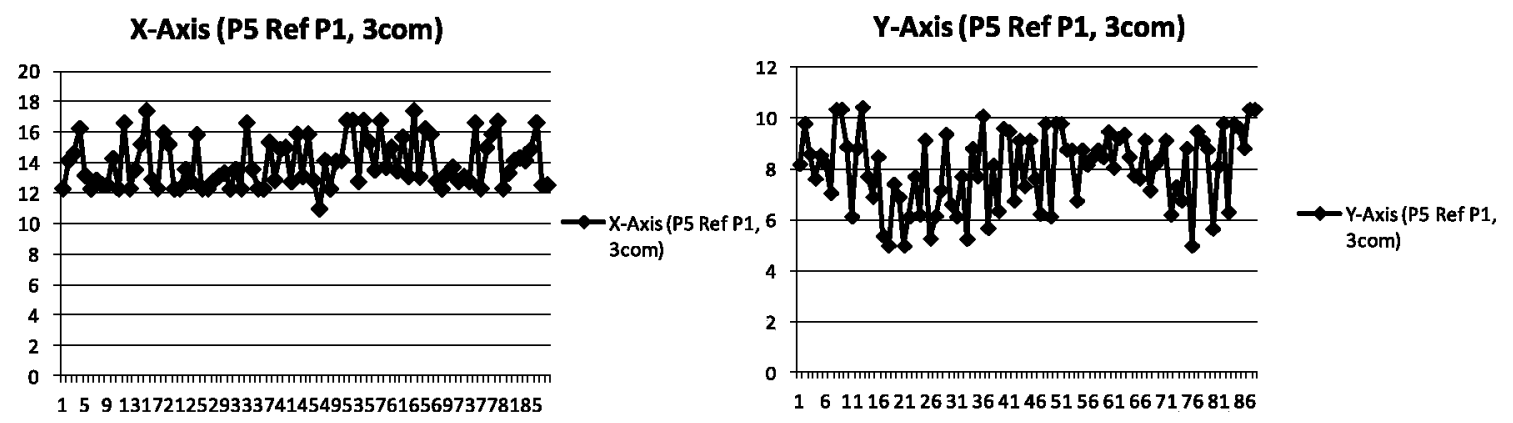

Fig. 19a. Coordinates $X, Y$ Fuzzy Controllers P5 (3com).
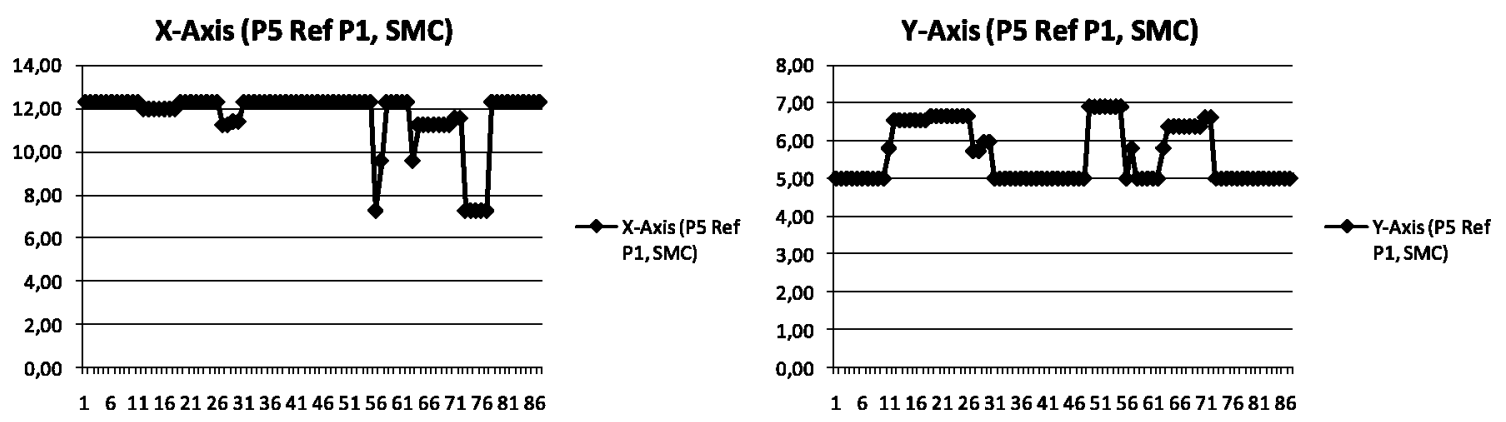

Fig. 19b. Coordinates $X, Y$ Fuzzy Controllers P5 (SMC).
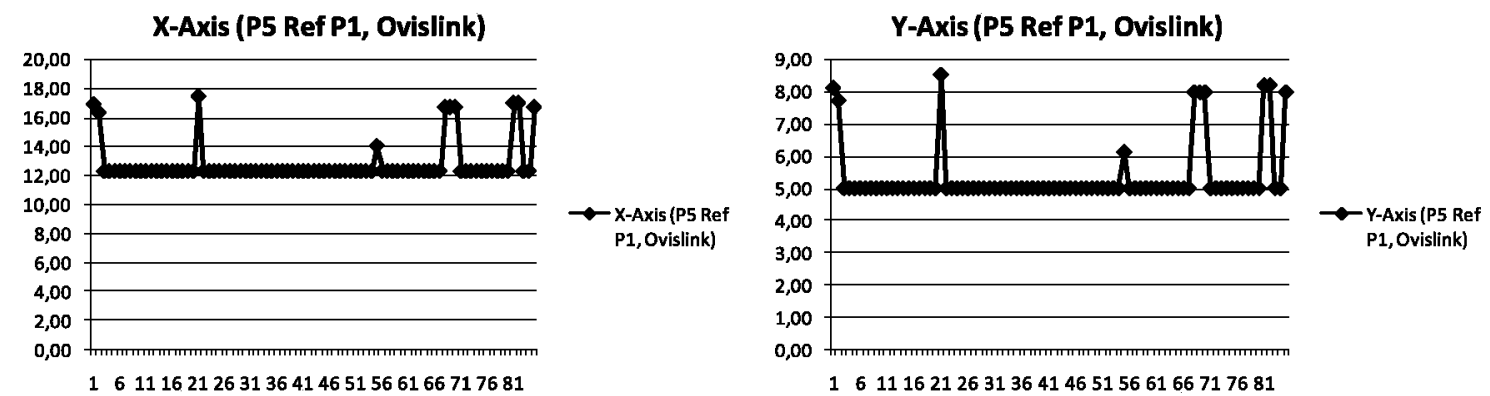

Fig. 19c. Coordinates $X, Y$ Fuzzy Controllers P5 (Ovislink).

X-Axis (P5 Ref P1, Robotics)

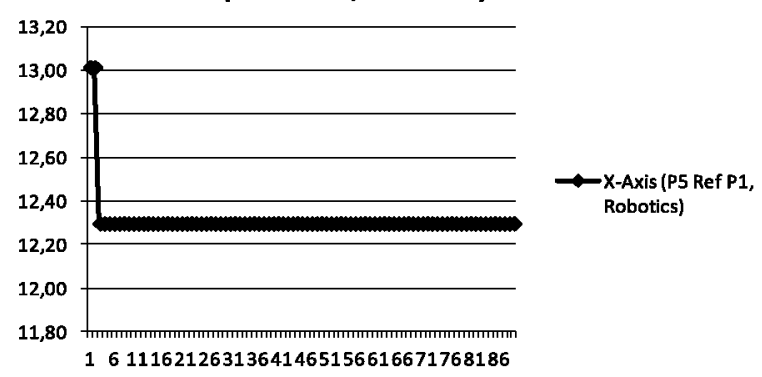

Y-Axis (P5 Ref P1, Robotics)

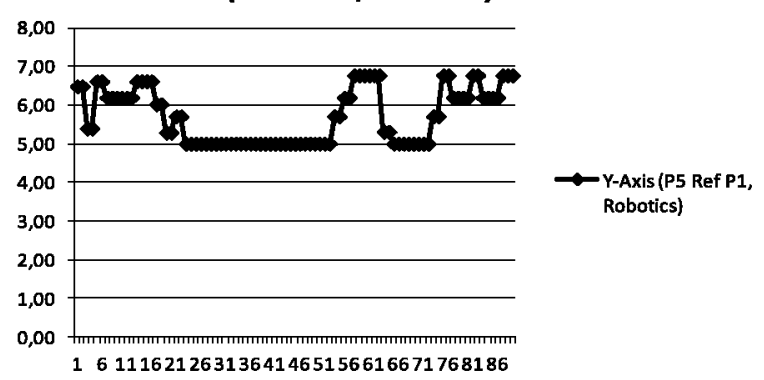

Fig. 19d. Coordinates $X, Y$ Fuzzy Controllers P5 (Robotics).

ers received from different MSs (see Tables $2 a-2 d$ ) provided with different wireless at the location point $\mathrm{P} 1$ :

It can be deduced from the analysis of these measurements that in accordance with the geometry of the layout of the access points and the thickness and quality of the walls on the floor being analyzed, it can be extrapolated that in the places close to the locations of the access points a pattern of RSSI measurements can be found that complies with any wireless interface whenever we normalize the measurements in respect to the power received from the closest access point.

The result is that we can place an MS in the area close to the access point by analyzing the pattern of power received. In the case of place P1, close to access point AP3 the normalized pattern in respect to the $\mathrm{AP}$ of greatest received power would be $\left(1,+2,{ }^{*},+2\right)$ these values having the meaning explained in Section 4.2 . 

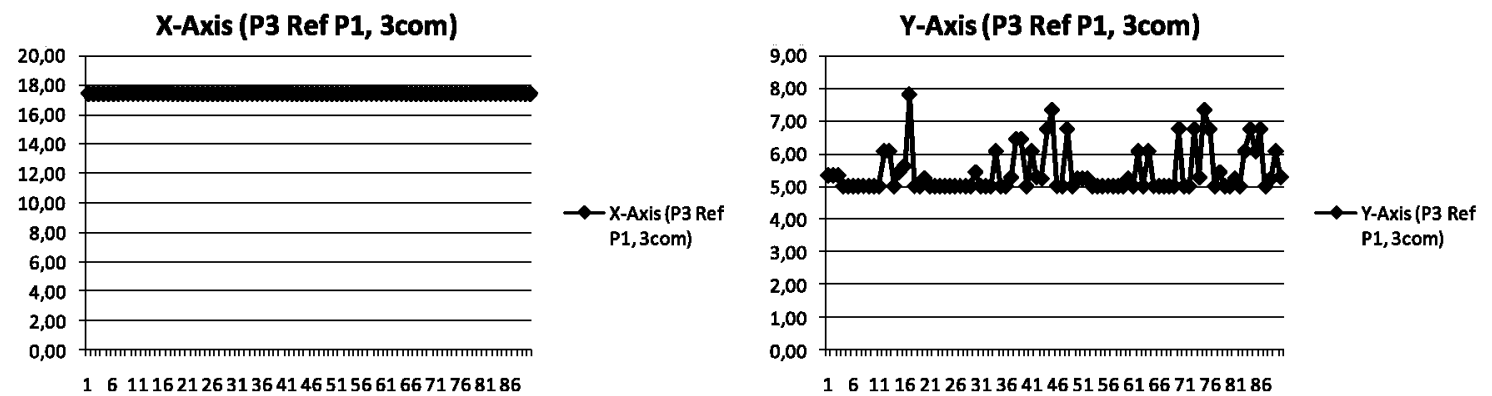

Fig. 20a. Coordinates $X, Y$ Fuzzy Controllers P3 (3com).
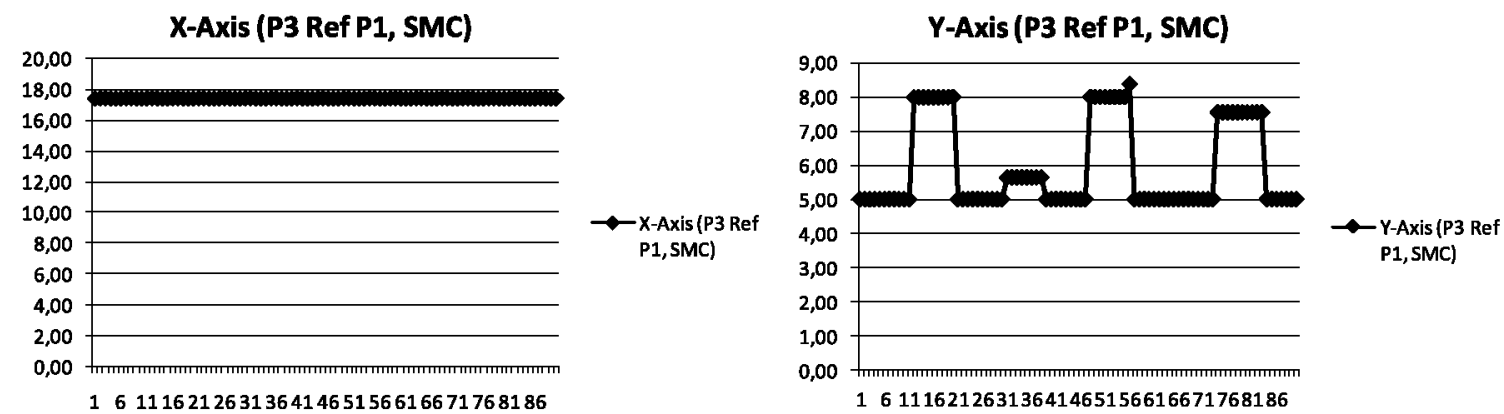

Fig. 20b. Coordinates $X$, Y Fuzzy Controllers P3 (SMC).
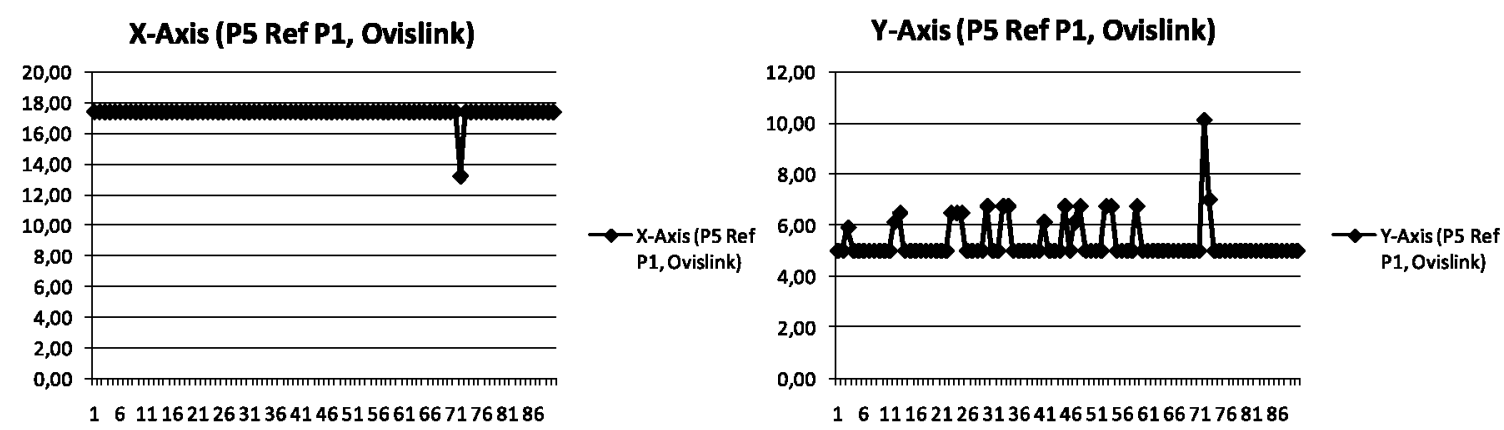

P1, Ovislink)

Fig. 20c. Coordinates $X, Y$ Fuzzy Controllers P3 (Ovislink).

X-Axis (P3 Ref P1, Robotics)

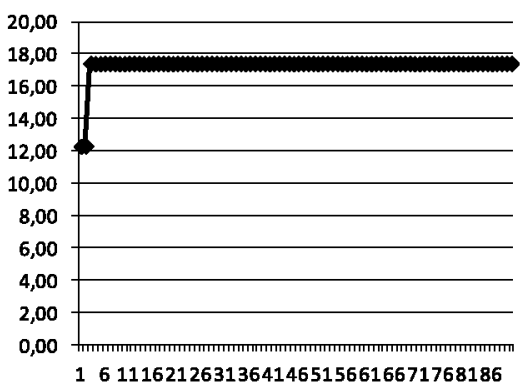

Y-Axis (P3 Ref P1, Robotics)

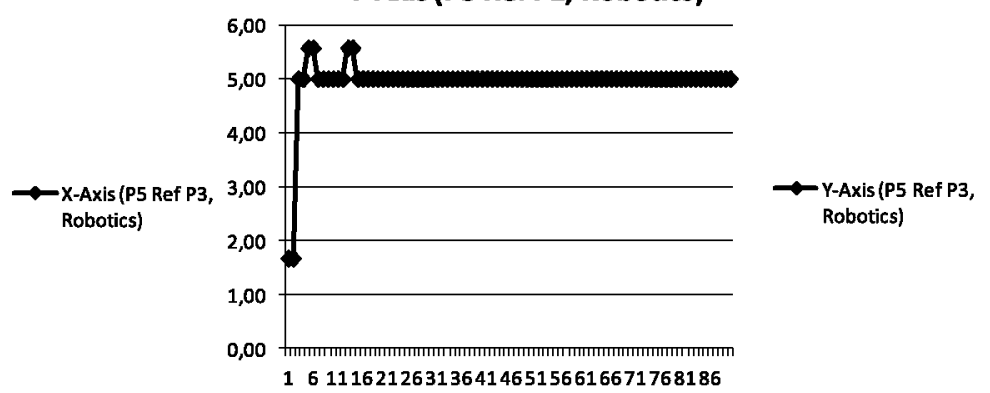

Fig. 20d. Coordinates $X, Y$ Fuzzy Controllers P3 (Robotics).

Note that the increase/decrease in power received from the AP1 normalized in respect to AP3 (which is the AP that emits the greatest power) is at any Wi-Fi interface greater than $20 \mathrm{~dB}$. This means a mask value of " +2 ".

The increase/decrease in power received from the AP2 normalized in respect to AP3 (which is the AP that emits the greatest power) is at any Wi-Fi interface between 10 and $20 \mathrm{~dB}$. This means a mask value of " +1 ".

Finally, the increase/decrease in power received from the AP4 normalized in respect to AP3 (which is the AP that emits the greatest power) is at any $\mathrm{Wi}-\mathrm{Fi}$ interface greater than $20 \mathrm{~dB}$. This means a mask value of " +2 ". 


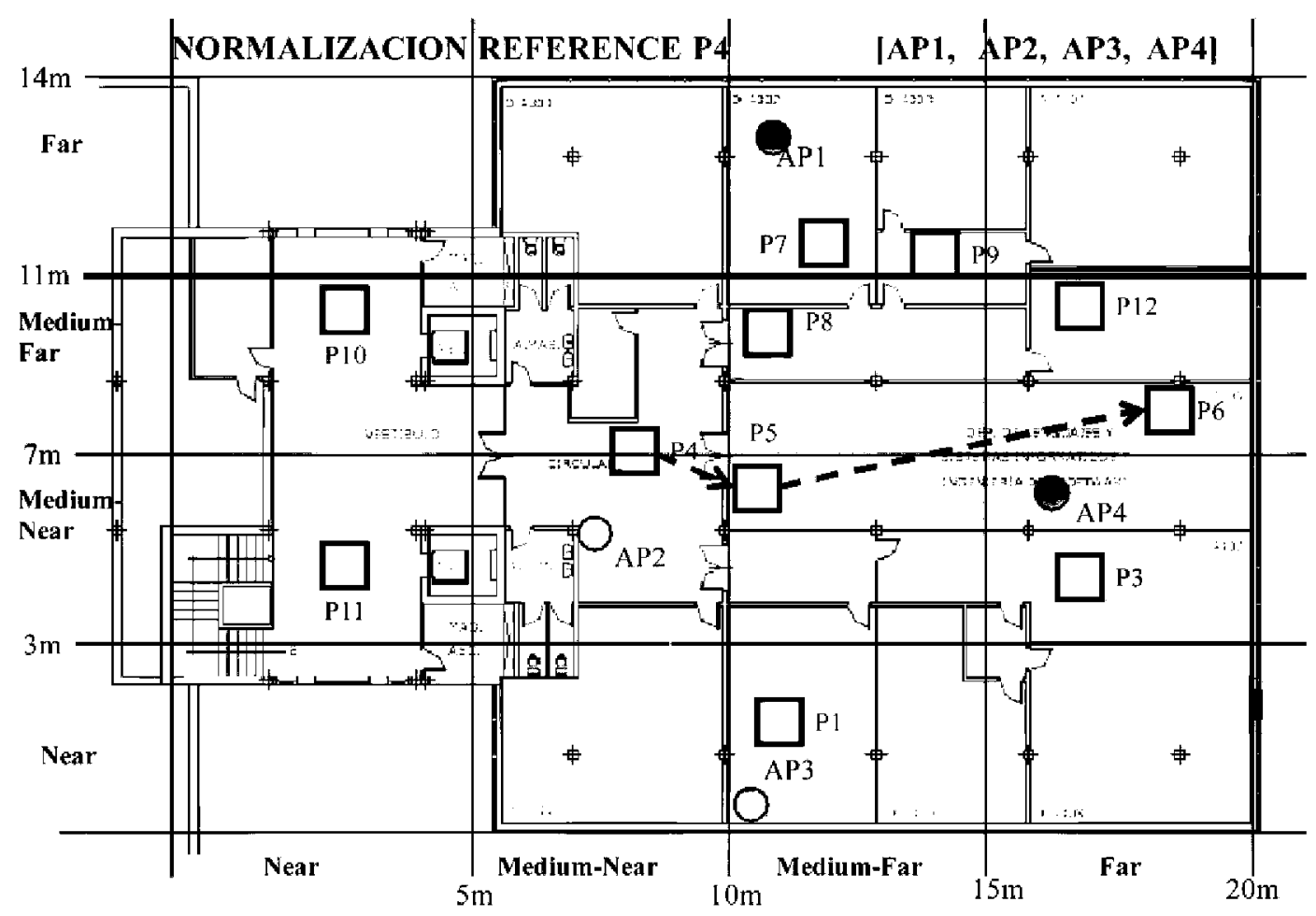

Fig. 21. Test 2. Movement of the MS.

The FLMSA carries out an initial estimation close to the position of the MS as if it were in a place close to the locations of the APs.

As a consequence of the results obtained, we propose that the APs are situated in a passing area of the MS so that the location is carried out optimally.

\subsection{Experiment 1: $P 1-P 5-P 3$ route}

In these experimental tests we work under the hypothesis that a mobile device (Mobile telephone, Smartphone, PDA or Laptop, or a Robotic system) has wireless technology and a Fuzzy Location Software Agent (FLSA).

In this first experiment we analyze the route of an MS from location P1 to P3 passing by P5. The FLSA of the MS takes a measurement of the power received from the APs on the floor (sent previously by the FLMSA). The FLMSA receives these power values and establishes that the position of the MS is in a location close to AP1. The FLMSA establishes a reference radio-map as that corresponding to location P1 (see Fig. 18). Remember that these Radio Maps were drawn up by following Algorithm 1.

The MS begins to move and arrives at position 5 . At this point the FLSA of the MS evaluates the power of the surrounding nodes and sends it to the FLMSA. This Agent is in charge of normalizing the measurements to be applied to the fuzzy controller under the rules corresponding to the Radio Map reference P1. By taking its reference from this place and the Radio Map for the reference point AP1 its position will be established using Algorithm 2 and we obtain the final position of the MS. The values obtained from the $X$ and $Y$ coordinates of the position of the MS on the map of the floor by the Fuzzy Controllers are detailed in Fig. 19a-19d for the different wireless interfaces used.

Bear in mind that the real situation of the MS would be between the values of axis $x$ between 10 and $15 \mathrm{~m}$ and the values of axis $Y$ between 3 and $7 \mathrm{~m}$. Note that there is no appreciable difference in the results obtained with the fuzzy controller using wireless cards from a different manufacturer.
The MS continues to move and arrives at a place identified by P3. In this place FLSA of the MS receives the power from the surrounding access points and sends this record to the FLMSA which will remember that the MS terminal passed by P1 and, by taking its reference at this point and the Radio Map for the reference point P1, it will establish its position using Algorithm 2. The values obtained from the $X$ and $Y$ coordinates of the position of the MS on the plan of the floor by the Fuzzy Controllers are set out in Fig. 20a-20d for the different wireless interfaces used.

Note that both in the case of the location P5 and location P3 the values obtained are independent of the Wi-Fi interface and it allows us to locate the MS in the current or immediate room.

Bear in mind that the real situation of the MS would be between the values of axis $x$ between 15 and $20 \mathrm{~m}$ and the values of axis $Y$ between 3 and $7 \mathrm{~m}$. Note that there is no appreciable difference in the results obtained with the fuzzy controller using wireless cards from a different manufacturer.

The fuzzy controller we have presented also incorporates an additional rule that is triggered when the measures of signal strength do not correspond to any location in the floor calculated using the rules built by means of the experimental study. The activation ratio of this rule in the proposed example lays between $5 \%$ and $10 \%$ of the measures taken.

\subsection{Experiment 2: P4-P5-P6 route}

In this second experiment an MS (Mobile station) is going to follow a route that corresponds to the places specified in Fig. 21 as P4-P5-P6.

The FLSA of the MS takes a measurement of the power received from the APs on the floor (sent previously by the FLMSA). The FLMSA receives these power values and establishes that the position of the MS is in a location close to AP2. The FLMSA establishes a reference radio-map as that corresponding to location P4 (see Fig. 22). Remember that these Radio Maps were drawn up by following Algorithm 1. 


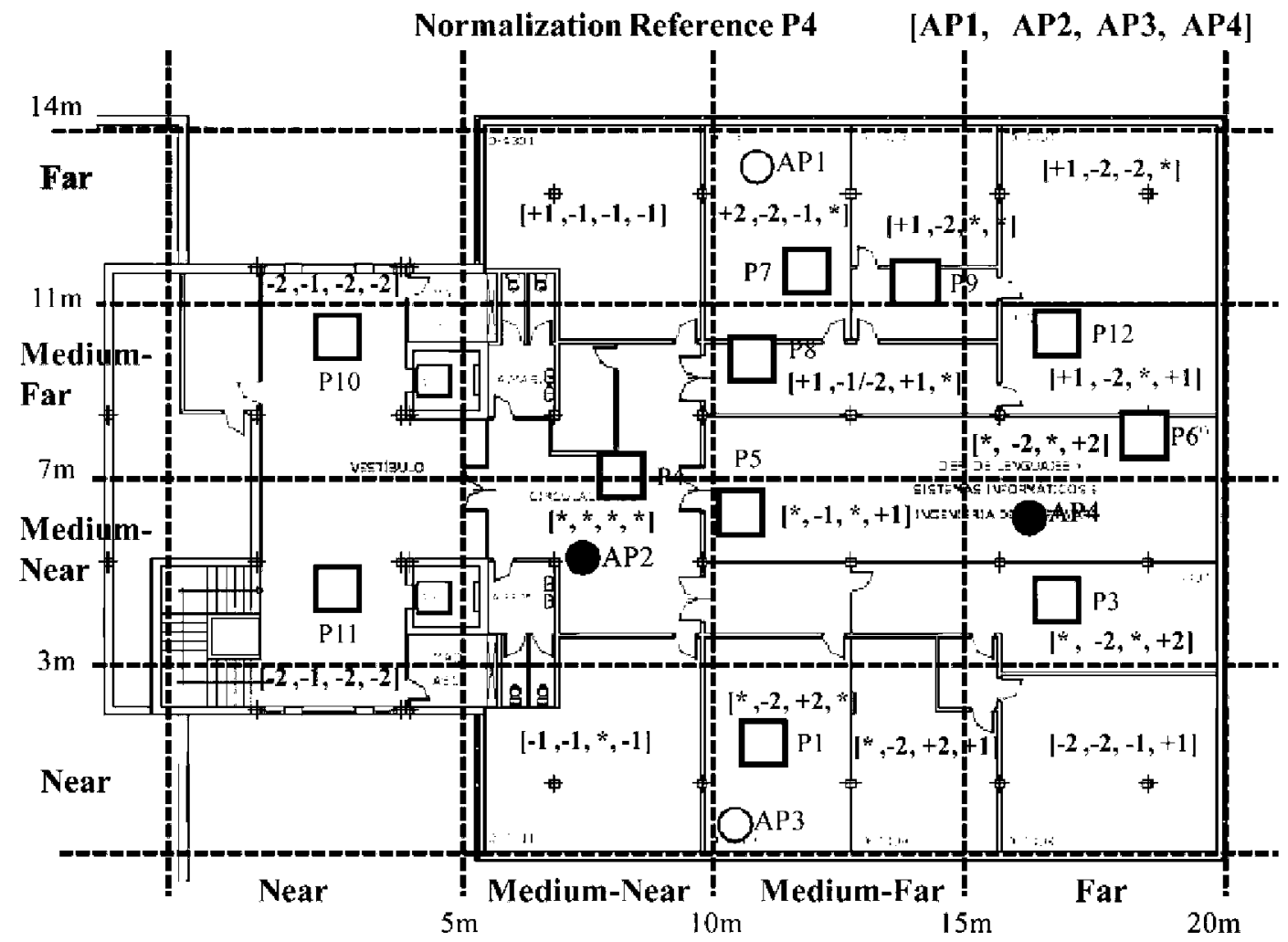

Fig. 22. Normalized measurements from location P4.
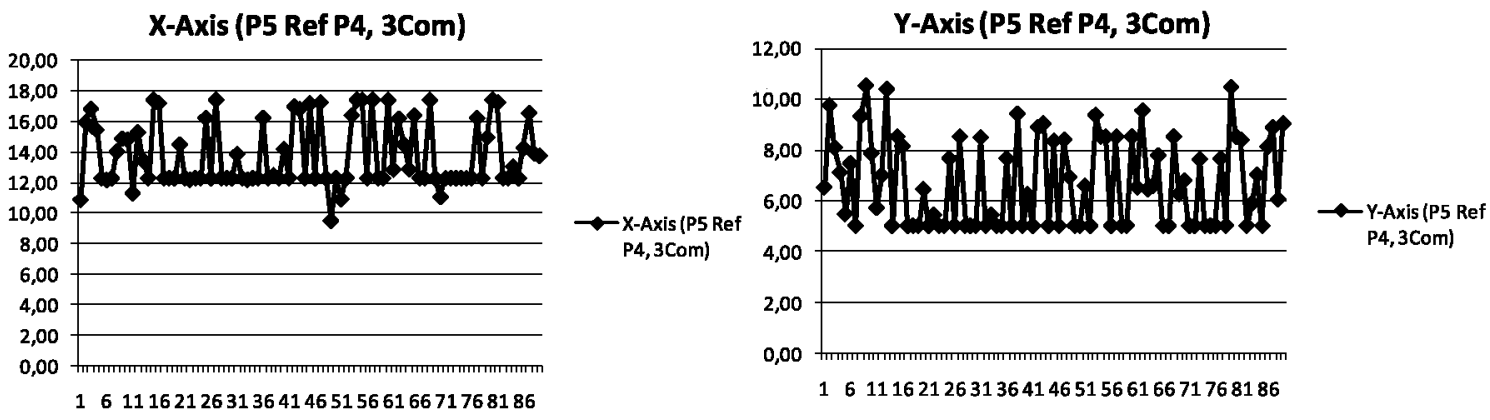

Fig. 23a. Coordinates $X, Y$ Fuzzy Controllers P5 (3com).
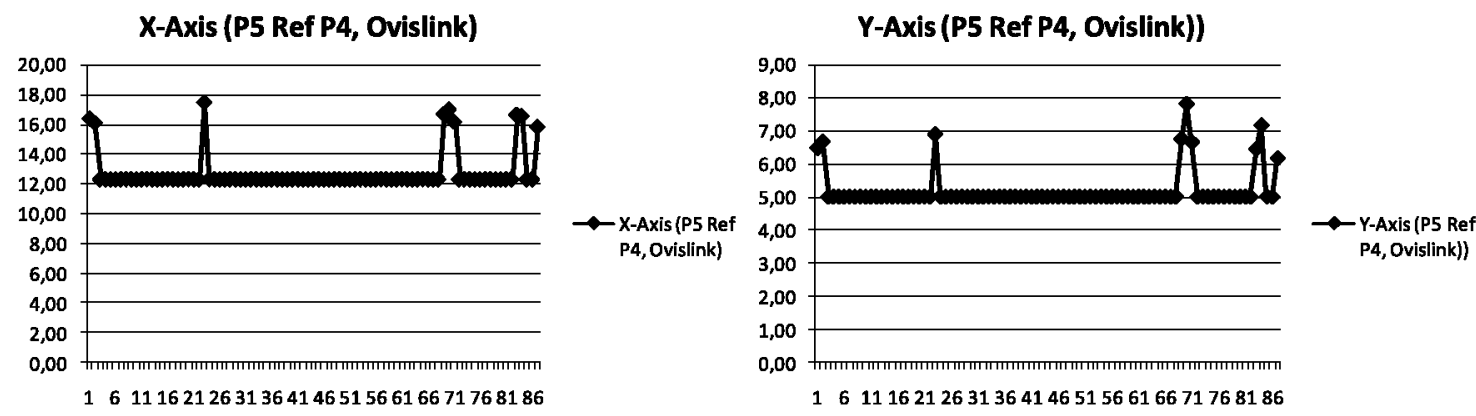

Fig. 23b. Coordinates $X$, Y Fuzzy Controllers P5 (Ovislink).

The MS continues to move and arrives at a place identified by P5. In this place FLSA of the MS evaluates the power from the surrounding access points and sends it to the FLMSA. This agent is in charge of normalizing the measurements and applying the fuzzy controller with the rules corresponding to the Radio Map reference P4. Taking its reference at this point and the Radio Map for the reference point AP2 it will establish its position using Algorithm 2, obtaining the final position of the MS. The values obtained from 
X-Axis (P6 Ref P4, 3Com)

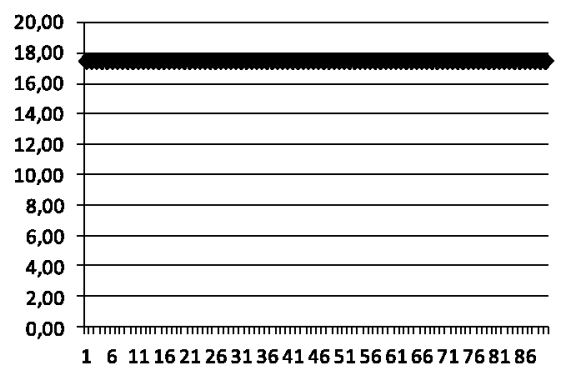

Y-Axis (P6 Ref P4, 3Com)

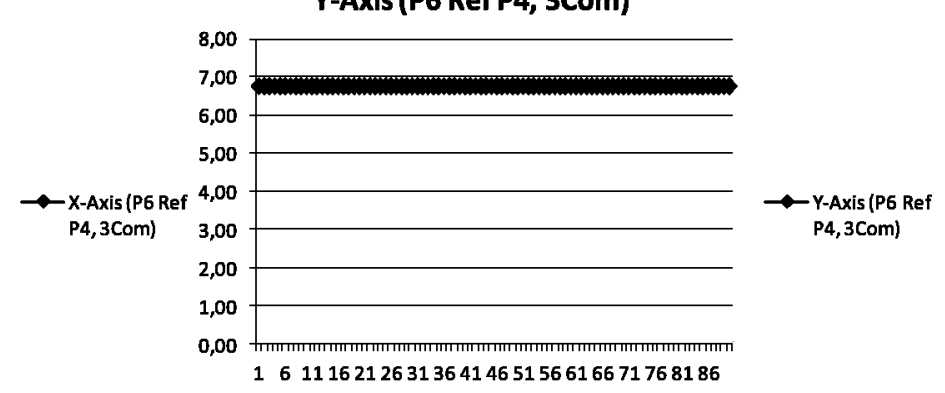

Fig. 24a. Coordinates $X, Y$ Fuzzy Controllers P5 (3Com).

X-Axis (P6 Ref P4, Ovislink)

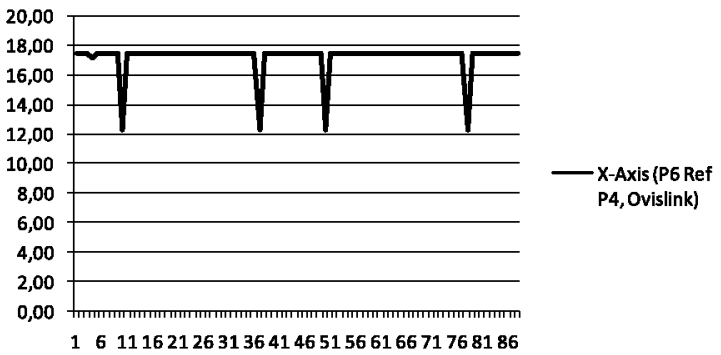

Y-Axis (P6 Ref P4, Ovislink)

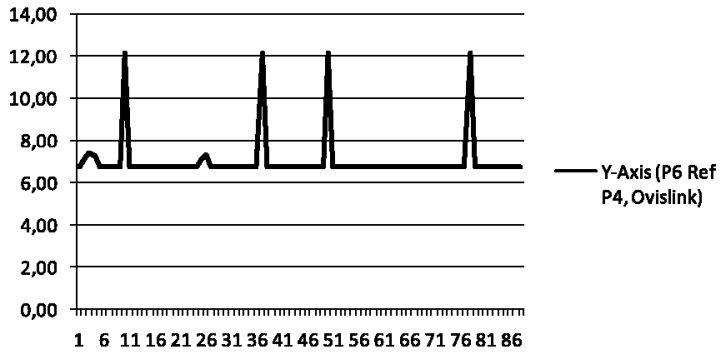

Fig. 24b. Coordinates $X, Y$ Fuzzy Controllers P5 (Ovislink).

the $X$ and $Y$ coordinates of the position of the MS on the plan of the floor by the Fuzzy Controllers are set out in Fig. 23a and 23b for the different wireless interfaces used.

Bear in mind that the real situation of the MS would be between the values of axis $x$ between 10 and $15 \mathrm{~m}$ and the values of axis $Y$ between 3 and $7 \mathrm{~m}$. Note that there is no appreciable difference in the results obtained with the fuzzy controller using wireless cards from a different manufacturer.

The MS continues to move and arrives at a place identified by P6. In this place FLSA of the MS receives the power from the surrounding access points and sends this record to the FLMSA which will remember that the MS terminal passed by $\mathrm{P} 4$ and, by taking its reference at this point and the Radio Map for the reference point P4, it will establish its position using Algorithm 2. The values obtained from the $X$ and $Y$ coordinates of the position of the MS on the plan of the floor by the Fuzzy Controllers are set out in Fig. 24a and 24b for any two wireless interfaces used.

Bear in mind that the real situation of the MS would be between the values of axis $x$ between 10 and $15 \mathrm{~m}$ and the values of axis $Y$ between 7 and $11 \mathrm{~m}$. Note that there is no appreciable difference in the results obtained with the fuzzy controller using wireless cards from a different manufacturer.

The fuzzy controller we have presented also incorporates an additional rule that is triggered when the measures of signal strength do not correspond to any location in the floor calculated using the rules built by means of the experimental study. The activation ratio of this rule in the proposed example lays between $5 \%$ and $8 \%$ of the measures taken.

\section{Conclusions}

We conceived and developed a system that is highly likely to estimate correctly the location of a mobile terminal indoors in a room or adjacent rooms. Unlike many other implemented systems, the resulting system is independent of the brand of the card and hardware technology (USB, PCMCIA) manufacturer. Our system evaluates the position by relative differences in the measurements taken at places close to the APs. The system is also adaptable to any environment. All it takes is system training with any card at locations on the floor, the normalization of the measurements and the creation of clusters. As of this point, the system is ready to locate any user with any wireless card.

The system developed is easily adaptable to new mobile devices: Smartphone, tables with $3 \mathrm{G}$ and $4 \mathrm{G}$ technologies, Wi-Fi by means of the integration of the FLMA software agent. The GSP location system of the MS in a building is now complemented by its exact location in a room on the floor of this building.

\section{References}

Abdelhadi, M., \& Anan, M. (2012). A fuzzy-based collaborative localization algorithm for wireless sensor networks. In International conference on collaboration technologies and systems (CTS) (pp: 152-156).

Ali, S., \& Nobles, P. (2007). A novel indoor location sensing mechanism for IEEE $802.11 \mathrm{~b} / \mathrm{g}$ wireless lan. In 4 th Workshop on positioning, navigation and communication, WPNC '07. March 2007. (pp. 9-15).

Bo-Chieh, L., \& Ken-Huang, L. (2008). Wireless location uses geometrical transformation method with single propagation delay: Model and detection performance. IEEE Transactions on Vehicular Technology, 57(5), 2920-2932.

Chai, X., \& Yang, Q. (2007). Reducing the calibration effort for probabilistic indoor location estimation. IEEE Transactions on Mobile Computing, 6(6), 649-662.

Chung, T.-Y., Chen, Y.-M., \& Tang, S.-C. (2012). A hybrid system integrating signal analysis and probabilistic neural network for user motion detection in wireless networks. Expert Systems with Applications, 39(3), 3392-3403.

Chun-Yu, S., Lyu-Han, C., Gen-Huey, C., Wu, E. H. -K., \& Ming-Hui, J. (2012) Intelligent radio map management for future WLAN indoor location fingerprinting. In IEEE Wireless communications and networking conference (WCNC) (pp 2769-2773).

Del Mundo, L.B., Ansay, R.L.D., Festin, C.A.M., \& Ocampo, R.M. (2011). A comparison of Wireless Fidelity (Wi-Fi) fingerprinting techniques. In International conference on ICT convergence (pp. 20-25 0), ICTC 2011. Art. No. 6082543.

Figuera, C., Rojo-Âlvarez, J. L., Wilby, M., Mora-Jiménez, I., \& Caamaño, A. J. (2012). Advanced support vector machines for 802.11 indoor location. Signal Processing, 92(9), 2126-2136.

Genming, D., Zhenhui, T., Lingwen, Z., Ziqi, Z., \& Jinbao, Z. (2012). Hybrid TOA/AOA cooperative localization in non-line-of-sight environments. In IEEE 75 th Vehicular technology conference (VTC Spring) (pp. 1-5).

IBM $^{\circledR}$ Rational $^{\circledR}$ SDL Suite ${ }^{\mathrm{TM}}$ (IBM Academic Initiative Program). http://www01.ibm.com/software/awdtools/sdlsuite/ 
Junyang, S., Molisch, A. F., \& Salmi, J. (2012). Accurate passive location estimation using TOA measurements. IEEE Transactions on Wireless Communications, 11(6), 2182-2192.

Kaemarungsi, K., \& Krishnamurthy, P. (2012). Analysis of WLAN's received signal strength indication for indoor location fingerprinting. Pervasive and Mobile Computing, 8(2). 292-3160

Mengual, L., Marbán, O., \& Eibe, S. (2010). Clustering-based location in wireless networks. Expert Systems with Applications, 37(9), 6165-6175.

Sawada, H., Takahashi, S., \& Kato, S. (2012). Disconnection probability improvement by using artificial multi reflectors for millimeter-wave indoor wireless communications. In IEEE 75th Vehicular technology conference (VTC Spring) (pp. 1-5).
SDL, Specification and description language ITU-T Rec. Z.100 (11/99). <http:// www.itu.int/ITU-T/studygroups/com10/languages/Z.100_1199.pdf>.

Shum, K. C. Y., Ng, J. K. -Y., \& Quan, J. C. (2012). The design and implementation of a wireless location estimation system in a wireless local area network. In 26 th International conference on advanced information networking and applications workshops (WAINA) (pp. 623-628).

Soria, L., Ortega, J., Alvarez, J., \& Gonzalez-Abril, L. (2012). Outdoor exit detection using combined techniques to increase GPS efficiency. Expert Systems with Applications, 39(15), 12260-12267.

Zietek, P., Kolakowski, J., \& Modelski, J. (2012). Investigation of the improved method for TDOA estimation with chirp signals. In 19th International conference on microwave radar and, wireless communications (MIKON) (pp. 377-380). 